\title{
Enumerating Gribov copies on the lattice
}

\author{
Ciaran Hughes $^{a *}$, Dhagash Mehta ${ }^{a, b}$, Jon-Ivar Skullerud ${ }^{a}$ \\ ${ }^{a}$ Department of Mathematical Physics, National University of Ireland Maynooth, \\ Maynooth, Co Kildare, Ireland \\ ${ }^{b}$ Department of Physics, Syracuse University, Syracuse, NY 13244, USA
}

February 6, 2013

\begin{abstract}
In the modern formulation of lattice gauge-fixing, the gauge fixing condition is written in terms of the minima or stationary points (collectively called solutions) of a gauge-fixing functional. Due to the non-linearity of this functional, it usually has many solutions called Gribov copies. The dependence of the number of Gribov copies, $n[U]$ on the different gauge orbits plays an important role in constructing the Faddeev-Popov procedure and hence in realising the BRST symmetry on the lattice. Here, we initiate a study of counting $n[U]$ for different orbits using three complimentary methods: 1 . analytical results in lower dimensions, and some lower bounds on $n[U]$ in higher dimensions, 2. the numerical polynomial homotopy continuation method, which numerically finds all Gribov copies for a given orbit for small lattices, and 3. numerical minimisation ("brute force"), which finds many distinct Gribov copies, but not necessarily all. Because $n$ for the coset $\mathrm{SU}\left(N_{c}\right) / \mathrm{U}(1)$ of an $\mathrm{SU}\left(N_{c}\right)$ theory is orbit-independent, we concentrate on the residual compact $\mathrm{U}(1)$ case in this article and establish that $n$ is orbit-dependent for the minimal lattice Landau gauge and orbit-independent for the absolute lattice Landau gauge. We also observe that contrary to a previous claim, $n$ is not exponentially suppressed for the recently proposed stereographic lattice Landau gauge compared to the naive gauge in more than one dimension.
\end{abstract}

\section{Introduction}

Though quantum field theory with the perturbative approach has been extremely successful, there are many important physical phenomena, such as quark confinement and dynamical chiral symmetry breaking in quantum chromodynamics (QCD), which need a non-perturbative treatment. To understand such a non-perturbative phenomenon, a genuine non-perturbative approach to QCD is essential. An immensely useful approach to study such non-perturbative

${ }^{*}$ Current address: Department of Applied Mathematics and Theoretical Physics, University of Cambridge, Cambridge, CB3 0WA, UK 
phenomena is lattice field theory [1, 2, 3. In this approach, the Euclidean space-time is discretised, so the four-dimensional space-time integral is replaced by a discrete sum over all lattice points while derivatives are replaced by finite differences. Then, Monte Carlo methods used in statistical mechanics can be applied to calculate the expectation values of observables.

In the continuum, a promising approach to study non-perturbative phenomena in QCD is to study truncated systems of Dyson-Schwinger equations (DSEs) [4, which are the equations of motion for QCD Green's functions. Since each gauge configuration comes with infinitely many equivalent physical copies, the set of which is called a gauge-orbit of the gauge configuration, the generating functional and hence any relevant quantity requires gauge-fixing to remove redundant degrees of gauge freedom.

The DSE approach has a clear advantage in the low momentum region of QCD. However, lattice QCD provides an opportunity to do first principles calculations of non-perturbative quantities in QCD: the approximations involved in lattice QCD can be systematically removed, unlike the truncations of DSEs. Thus, lattice simulations can provide an independent check on the results obtained in the DSE approach. A gauge field theory put on the lattice is manifestly gauge invariant, i.e., one does not need to fix a gauge on the lattice to calculate gauge invariant observables. However, to compare with DSE results, gauge fixing is necessary, and it is mainly for this reason that lattice Landau gauge studies have gained a large amount of interest recently. The standard approach of gauge-fixing in the perturbative limit is the Faddeev-Popov (FP) procedure [5]. There, a gauge-fixing device which is called the gauge-fixing partition function, $Z_{G F}$, is formulated. With an ideal gauge-fixing condition, $Z_{G F}$ is equal to one. This unity is inserted in the measure of the generating functional so that the redundant degrees of freedom are removed after appropriate integration. The generalisation of the FP procedure is the Becchi-Rouet-Stora-Tyutin (BRST) formulation [6]. The assumption that the gauge-fixing condition is ideal, i.e., the gauge-fixing condition has a unique solution, is crucial here. However, V. N. Gribov, in 1978, found that in non-Abelian gauge theories a generalised Landau gauge-fixing condition treated non-perturbatively would have multiple solutions, called Gribov or Gribov-Singer copies [7, 8, 4, the effects of which should be properly taken into account.

There is one more obstacle on the lattice. If a procedure analogous to the FP procedure is carried out on the lattice, the lattice analogue of $Z_{G F}$ turns out to be zero 9, 10] due to a perfect cancellation among Gribov copies 1 . Thus, the expectation value of a gauge-fixed observable turns out to be of the indeterminate form $0 / 0$. This problem is known as the Neuberger $0 / 0$ problem. In other words, BRST formulations on the lattice can not be constructed. This severely hampers any comparison with continuum DSE studies [14, 15, 16.

On the lattice, Landau gauge fixing is usually formulated as a functional minimisation problem. That is, instead of solving the lattice counterpart of the gauge-fixing conditions, one numerically minimises a gauge-fixing functional, whose first derivative with respect to gauge transformation is the lattice counterpart of the gauge-fixing condition. The space of minima, called the first Gribov region, also has many Gribov copies but there is no cancellation among these Gribov copies hence the Neuberger $0 / 0$ problem is avoided. However, the number of minima may be different for different gauge-orbits making the corresponding $Z_{G F}$ orbit-dependent. In this case, inserting $Z_{G F}$ in the partition function becomes quite cumbersome. It is this orbit-dependence or orbit-independence of the $Z_{G F}$ in various versions of lattice Landau gauge which is the main

\footnotetext{
${ }^{1}$ See Refs. [11, 12 13, for earlier attempts.
} 
focus of this paper.

Gribov copies not only play an important role in constructing the BRST symmetry on the lattice, but it is also extensively argued that they influence the infrared behaviour of the gauge dependent propagators of gauge theories (See, e.g., [17, 18, 19, and 20, 21, 22, 23, for a continuum perspective).

This paper is organised as follows. In Section 2 we introduce gauge-fixing on the lattice, fix the notation and set up the general problem. We also introduce several versions of lattice Landau gauge which we will study in the remainder of the paper. In Section 3 we formulate the gauge fixing problem for the compact $\mathrm{U}(1)$ case. We also summarise the previously known analytical results in one dimension and work out some analytical results in higher dimensions. In Section 4, we introduce a novel numerical method which finds all the stationary points of a given potential provided that the corresponding stationary equations are polynomial-like and have only finitely many solutions. We show how to translate the lattice Landau gauge-fixing equations for the compact $U(1)$ case into a system of polynomial equations, and then find all the Gribov copies for small but non-trivial lattices. Section 5 contains the results from our numerical minimisation of the gauge fixing functional. Finally, in Section [6 we summarise our main results and discuss the implications of our findings.

\section{Gauge-fixing on the lattice}

A gauge field theory can be studied nonperturbatively by discretising the Euclidean space-time and putting the gauge and matter fields of the theory on a four-dimensional space-time grid 1 . The gauge fields are usually defined through link variables $U_{i, \mu} \in G$ where the discrete variable $i$ denotes the site index, $\mu$ is a directional index and $G$ is the corresponding group of the theory. The relation of the gauge-fields on the lattice $U_{i, \mu} \equiv U_{\mu}(x)$ to their continuum counterpart $A_{\mu}(x)$, is given by

$$
U_{\mu}(x)=\mathcal{P} \exp \left[i \int_{x}^{x+\hat{\mu}} A_{\mu}(\xi) d \xi\right]
$$

where $\mathcal{P}$ denotes path ordering.

The lattice expectation value of an observable $O$ is

$$
\langle O\rangle=\frac{\int \prod_{i, \mu} d U_{i, \mu} \exp [-S[U]] O\left[U_{i, \mu}\right]}{\int \prod_{i, \mu} d U_{i, \mu} \exp [-S[U]]},
$$

where $S[U]$ is the lattice action.

The action $S[U]$ is invariant under $U_{i, \mu} \rightarrow U_{i, \mu}^{g}=g_{i}^{\dagger} U_{i, \mu} g_{i+\hat{\mu}}$, where the gauge transformations $g_{i} \in G$ are defined at each lattice site. In other words, a set of randomly chosen $\{U\}$ comes with infinitely many physically equivalent configurations the set of which is called a gauge orbit and represented by the set $\{U\}$. This gauge invariance makes the integration in the continuum counterpart of Eq. (2) ill-defined. In the continuum, to get rid of this ambiguity, one can choose to take exactly one representative (or more than one but a finite number) of each gauge-orbit. This is called gauge fixing. 
Choosing the representatives of each gauge-orbit can in practice be done by imposing a constraint of the form $f\left(U^{g}\right)=0$ in Eq. (2). One takes the integral over the gauge orbit, $U^{g}$,

$$
n[U] \equiv \int d g \operatorname{det} M_{F P}(g) \delta\left(f\left(U^{g}\right)\right)=: Z_{G F} .
$$

where $Z_{G F}$ is called the gauge fixing partition function, and det $M_{F P}$ is the determinant of the Jacobian of the gauge-fixing condition, called the Faddeev-Popov (FP) determinant. Here, $n[U]$ may be a function of the gauge orbit $\{U\}$, that is, $n[U]=n\left[U^{g}\right]$. For non-Abelian theories treated nonperturbatively, the surface $f\left(U^{g}\right)=0$ will in general have more than one solution, called Gribov copies. If $n[U]$ is non-vanishing for all orbits, then inserting Eq.(3) in (2) gives, after factoring out the gauge volume,

$$
\langle O\rangle=\frac{\int \prod_{i, \mu} d U_{i, \mu} \frac{1}{n[U]} \operatorname{det} M_{F P}(g) \delta\left(f\left(U^{g}\right)\right) \exp [-S[U]] O\left[U_{i, \mu}\right]}{\int \prod_{i, \mu} d U_{i, \mu} \frac{1}{n[U]} \operatorname{det} M_{F P}(g) \delta\left(f\left(U^{g}\right)\right) \exp [-S[U]]},
$$

provided that the operator $O[U]$ is gauge invariant. However, this expression can also be used for gauge dependent operators, in which case it defines the gauge fixed expectation value.

The most popular gauge is the Landau gauge, which can be formulated as solving the following conditions in the continuum,

$$
\partial_{\mu} A_{\mu}=0
$$

or as a functional minimisation problem [24] of the Landau gauge fixing functional

$$
F_{A}[g]=\left\|A^{g}\right\|^{2}=-\int d^{4} x \operatorname{Tr}\left(\left(g A_{\mu} g^{\dagger}\right)^{2}\right),
$$

whose first derivatives with respect to gauge parameters $g$ are shown to give Eq. (5). The Hessian matrix $M_{F P}$ of this functional is the FP operator.

The functional minimisation approach carries over straightforwardly to the lattice formulation. The standard choice of the lattice Landau gauge-fixing functional, in the following called the naive or direct lattice Landau gauge functional, to be minimised with respect to the corresponding gauge transformations $g_{i}$, is

$$
F_{U}(g)=\sum_{i, \mu}\left(1-\frac{1}{N_{c}} \operatorname{Re} \operatorname{Tr} g_{i}^{\dagger} U_{i, \mu} g_{i+\hat{\mu}}\right),
$$

for $\mathrm{SU}\left(N_{c}\right)$ groups. Taking $f_{i}(g)=\frac{\partial F_{U}(g)}{\partial g_{i}}=0$ for each lattice site $i$ gives the lattice divergence of the lattice gauge fields and in the naive continuum limit recovers the Landau gauge $\partial_{\mu} A_{\mu}=0$. The corresponding $M_{F P}$ is the Hessian matrix of $F_{U}(g)$, with respect to the gauge transformations.

Neuberger showed 9, 10, that when all the stationary points, not only minima, of the naive functional are taken into account, $Z_{G F}$ turns out to be zero and the expectation value of a gauge-fixed variable then is $0 / 0$. Schaden [25] interpreted the problem in terms of Morse theory and showed that $Z_{G F}$ calculates the Euler character $\chi$ of the group manifold $G$ at each site of the lattice. That is, for a lattice with $N$ lattice sites,

$$
Z_{G F}=\sum_{i} \operatorname{sign}\left(\operatorname{det} M_{F P}(g)\right)=(\chi(G))^{N},
$$


where the sum runs over all the Gribov copies. The group manifold $G$ for compact U(1) is a circle $S^{1}$ and for $\mathrm{SU}\left(N_{c}\right)$ it is $S^{3} \times S^{5} \times \cdots \times S^{2 N_{c}-1}$. This result is derived from the PoincaréHopf theorem which asserts that $\chi(\mathbb{M})$ is equal to the sum of signs of Hessian determinants at all critical points of a non-degenerate height function which is a compact, differentiable and orientable function from the manifold $\mathbb{M}$ to $\mathbb{R}$. In the case of lattice Landau gauge fixing we can immediately identify the gauge-fixing functional (7) as a height function, Gribov copies as the critical points and $M_{F P}$ as the corresponding Hessian matrix.

For compact U(1), $G=S^{1}$. As Eq. (77) can be viewed as a height function from $S^{1} \times S^{1} \cdots \times S^{1}$ to $\mathbb{R}$, and since $\chi\left(S^{1}\right)=0$, we have $Z_{G F}=0$. As we will explicitly see in section 3 , antiperiodic boundary conditions in this case fix the global gauge freedom, so the corresponding manifold is $\left(S^{1}\right)^{N}$. For periodic boundary conditions the corresponding manifold is $\left(S^{1}\right)^{N-1}$. Thus, for any boundary conditions, we have $Z_{G F}=0$. In fact, for any $\mathrm{SU}\left(N_{c}\right)$, and hence for the standard model group, the group manifolds are odd-dimensional spheres for which $\chi$ is zero.

Following this interpretation, for SU(2) gauge theory, Schaden proposed to construct a BRST formulation only for the coset space $\mathrm{SU}(2) / \mathrm{U}(1)$ for which $\chi \neq 0$ (the residual compact $\mathrm{U}(1)$ symmetry was left unaddressed). This procedure can be generalised to fix the gauge of an $\mathrm{SU}\left(N_{c}\right)$ lattice gauge theory to the maximal Abelian subgroup $(\mathrm{U}(1))^{N_{c}-1}$, since $\chi\left(\mathrm{SU}\left(N_{c}\right) / \mathrm{U}(1)\right) \neq 0$ as well. This indicates that the Neuberger $0 / 0$ problem for an $\mathrm{SU}\left(N_{c}\right)$ lattice gauge theory actually lies in $(\mathrm{U}(1))^{N_{c}-1}$, and hence can be evaded if the problem for compact $\mathrm{U}(1)$ is evaded. This is why we concentrate our study on the compact $U(1)$ case from now on.

We should emphasise here that there are no Gribov copies in continuum Quantum Electrodynamics (QED) which is a U(1) gauge theory. More specifically, on the lattice, the compactness of the gauge group introduces Gribov copies for the compact U(1) theory. Thus, Gribov copies on the lattice are purely lattice artefacts and so is the Neuberger 0/0 problem. As argued above, we are interested in studying Gribov copies of compact U(1) theory because the Neuberger 0/0 problem apparently lies in the residual compact U(1) subgroup. Having said that, we should also note that compact QED on the lattice may serve as a prototype for theories based on compact groups such as $\mathrm{SU}\left(N_{c}\right)$, and hence studying compact QED on the lattice is important in its own right. Compact QED in four dimensions exhibits two phases: a Coulomb phase, with a massless photon, and a confined phase, which, although unphysical, shares many qualitative features with QCD.

To avoid the Neuberger $0 / 0$ problem, one may modify the gauge fixing condition in some way. However, any such modified lattice gauge-fixing should satisfy the following conditions:

1. The corresponding $n[U]$ should be orbit-independent. If this is the case, then $n[U]$ cancels out in Eq. (4), otherwise it must be computed for each configuration, which is usually not feasible as it involves finding all solutions to $f_{i}\left(U^{g}\right)=0$, for $i$ running over all the lattice sites.

2. It should be possible to efficiently implement the corresponding gauge-fixing numerically.

3. The additional gauge-fixing terms should not destroy the theory, e.g., the gauge-fixed action should be renormalisable.

Below we discuss a few alternative gauge fixing procedures: 
Minimal lattice Landau gauge: Here, instead of taking all the stationary points of the gaugefixing functional, one only considers the space of minima, called the first Gribov region. Since $M_{F P}$ is positive definite for the minima, by definition, there is no cancellation among the signs of determinants of $M_{F P}$ (i.e., $n[U]$ is just the total number of local and global minima), and hence no Neuberger $0 / 0$ is present there. This is also advantageous numerically, since finding minima is easier than finding general stationary points. It has also been shown [26 that this restriction can be written in terms of a renormalisable action with auxiliary fields (for a review of this approach, see [27]). However, a crucial point is whether the corresponding $n[U]$ is orbit-independent. If it is orbit-dependent then each orbit comes with a different $n[U]$ and the functional integral becomes a function of $n[U]$ and cumbersome to deal with. In the one-dimensional case for compact $\mathrm{U}(1)$, it has already been shown that the corresponding $n[U]$ is orbit-dependent [28, 29]. In the present paper, one of our goals is to verify this in the two-dimensional case.

Absolute lattice Landau gauge: In this gauge, one further restricts the gauge-fields to the space of global minima, called the fundamental modular region (FMR). Here, in addition to evading the Neuberger $0 / 0$ problem by avoiding the cancellation among the determinants of $M_{F P}$ (i.e., $n[U]$ is nothing but the number of global minima), we also expect the corresponding $n[U]$ to be orbit-independent, and equal to 1 in the general case. It is also anticipated that the set of configurations with degenerate global minima is a set of measure zero which forms the boundary of the FMR. In other words, there are no Gribov copies inside the FMR [30, 31. In the onedimensional compact $\mathrm{U}(1)$ case, this was also verified to be true [28, 29. In the current paper, we want to study this issue in the two-dimensional case. Having said that, we emphasise that finding the global minimum of such functions (which corresponds to spin glass model Hamiltonians) is known to be a very difficult task and in most cases it is an NP hard problem. Thus, in realistic cases, we can not expect to find the global minimum using conventional numerical minimisation methods, and the best one may do is to generate a number of minima and choose the 'best minimum' among these as an approximation to the global minimum (see eg. [19, 32]). In addition to this, the absolute lattice Landau gauge can not be stated in terms of algebraic conditions, making it difficult to impose using the standard FP procedure. In this paper, we provide evidence that $n[U]$ for this gauge is indeed orbit-independent.

Stereographic lattice Landau gauge: A modification of the group manifold of compact $\mathrm{U}(1)$, i.e., a circle $S^{1}$, via stereographic projection at each lattice site was proposed and studied in Refs. 14, 15, 28. For such a stereographically projected manifold the corresponding $\chi$ is nonzero and thus the Neuberger $0 / 0$ problem is completely avoided. Applying the same technique to the maximal Abelian subgroup $(\mathrm{U}(1))^{N_{c}-1}$, the generalisation to $\mathrm{SU}\left(N_{c}\right)$ lattice gauge theories is possible when the odd-dimensional spheres $S^{2 k+1}, k=1, \ldots, N_{c}-1$, of its parameter space are stereographically projected to the real projective space $\mathbb{R} P(2 k)$. It was also shown using topological arguments that the number of Gribov copies is exponentially suppressed for the stereographic compared to the naive gauge for compact $\mathrm{U}(1)$, and that the corresponding $n[U]$ is orbit-independent, at least for the one-dimensional lattice case. It can be shown that the corresponding FP operator in this case is generically positive (semi-)definite (i.e., all stationary points are minima in this case, and there are no saddle points nor maxima) and hence $n[U]$ is nothing but the total number of local and global minima. The stereographic lattice Landau gauge is a promising alternative from the lattice BRST symmetry point of view since it fulfils all the above mentioned practical requirements, except that the orbit-independence is yet to be confirmed for lattices in more than one dimension. 
Interestingly, in lattice formulations of supersymmetric Yang-Mills theories, non-compact parameterisations (similar to the stereographic projection) of the gauge fields are used [33. The non-compact parametrisation, unlike the compact (group based) parametrisation, apparently evades the sign problem in the lattice versions of these supersymmetric theories 34, 35. Recently, a deeper and direct connection between the sign problem in lattice supersymmetric theories and the Neuberger $0 / 0$ problem has been established [36]: essentially, the complete action of $\mathcal{N}=2$ supersymmetric Yang-Mills theories in two dimensions can be shown to be a gauge-fixing action, analogous to the Faddeev-Popov procedure, with the symmetry now being a topological gauge symmetry. Thus, the corresponding partition function is nothing but $Z_{G F}$ for the corresponding theory and hence the Neuberger $0 / 0$ problem follows for the compact parametrisation. For the non-compact parametrisation, the Neuberger $0 / 0$ problem can be avoided due to topological arguments.

Other approaches and efforts: An alternative to fixing the gauge completely would be to average over Gribov copies, as proposed in Refs. [37, 38, 39], with a weight proportional to the $\exp \left(-\beta_{G F} F[g]\right)$, where $F[g]$ is the gauge fixing functional of (6) or (7). This reformulation evades the Neuberger problem. In the original formulation, the average was over the whole gauge orbit, and the (absolute) Landau gauge would only be reproduced in the limit $\beta \rightarrow \infty$. It has recently been shown [40] that if the average is taken only over configurations satisfying the Landau gauge condition, the resulting action is renormalisable and possesses a BRST-like symmetry. This class of gauges has been studied numerically [41; however, it is expensive as it amounts to performing a second Monte Carlo integral over Gribov copies on top of the usual lattice Monte Carlo sampling.

An orthogonal approach was recently proposed 42, in which the Gribov copies are treated as a residual, nonperturbative gauge degree of freedom which (at least in the continuum, infinite volume limit) can be fixed by imposing additional conditions on the correlation functions. For example, it is suggested that one may define a family of gauges, so-called Landau- $B$ gauges, by defining a target value $B$ for the renormalised ghost propagator at a specific momentum and choosing the Gribov copy that gives a result closest to this value. A particular choice is the max- $B$ gauge which selects the Gribov copy with the largest value for $B$. Whether or to what extent it is possible to impose such a condition is at present not clear, and little or nothing is as yet known about the relation between the $B$ parameter and the Landau gauge fixing functional.

Another way was put forward in Refs. 43, 44, 45] using the ghost/anti-ghost symmetric CurciFerrari gauges. There the argument used was that the Neuberger $0 / 0$ problem could be extended to include such non-linear gauges with their extended double-BRST symmetry despite their quartic ghost self-interactions, which allow the introduction of a mass term for ghosts. Such a Curci-Ferrari mass would break the nilpotency of the BRST/anti-BRST charges which is known to result in a loss of unitarity; however, this mass also serves to regulate the Neuberger zeroes in a lattice formulation and expectation values of observables can then be meaningfully defined in the limit $m \rightarrow 0$ via l'Hospital's rule.

On the continuum side, an explicit counting of Gribov copies was carried out in 20, 21]. There, the $\mathrm{SU}(2)$ case was considered, but restricted to static spherically symmetric configurations only (in which case Landau and Coulomb gauge conditions are identical).

This is not intended to be an exhaustive review of previous attempts to address this subject. For a pedagogical and thorough review of previous work on this topic, we refer to the recent review [46]. 


\section{Lattice Landau gauge for compact $\mathrm{U}(1)$}

Since the group manifold of compact $\mathrm{U}(1)$ is topologically a circle $S^{1}$, we can write the link variables and gauge transformations in terms of angles $\phi_{i, \mu}, \theta_{i} \in(-\pi, \pi] \bmod 2 \pi$, as $U_{i, \mu}=$ $e^{i \phi_{i, \mu}}$ and $g_{i}=e^{i \theta_{i}}$, respectively. Thus, the naive gauge fixing functional Eq. (7) is reduced to

$$
F_{\phi}(\theta)=\frac{1}{V} \sum_{i, \mu}\left(1-\cos \left(\phi_{i, \mu}+\theta_{i+\hat{\mu}}-\theta_{i}\right)\right) \equiv \frac{1}{V} \sum_{i, \mu}\left(1-\cos \phi_{i, \mu}^{\theta}\right),
$$

where we have defined $\phi_{i, \mu}^{\theta}:=\phi_{i, \mu}+\theta_{i+\hat{\mu}}-\theta_{i}$. Note that we have also introduced a normalisation factor $1 / V$, where $V$ is the lattice volume. The stereographic gauge fixing functional becomes

$$
F_{\phi}^{s}(\theta)=-\frac{2}{V} \sum_{i, \mu} \ln \left(\cos \left(\phi_{i, \mu}^{\theta} / 2\right)\right)
$$

A given random set of $\phi_{i, \mu}$ is called a random orbit or a hot configuration. The special case when all $\phi_{i, \mu}$ are zero is called the trivial orbit, or cold configuration. The gauge-fixing conditions are, respectively,

$$
\begin{aligned}
f_{i}(\theta) & =-\sum_{\mu=1}^{d}\left(\sin \phi_{i, \mu}^{\theta}-\sin \phi_{i-\hat{\mu}, \mu}^{\theta}\right)=0, \\
f_{i}^{s}(\theta) & =-\sum_{\mu=1}^{d}\left(\tan \left(\phi_{i, \mu}^{\theta} / 2\right)-\tan \left(\phi_{i-\hat{\mu}, \mu}^{\theta} / 2\right)\right)=0
\end{aligned}
$$

for all lattice sites $i$. The FP operator for the two gauges are

$$
\begin{aligned}
& \left(M_{F P}\right)_{i, j}=\sum_{\mu}\left(-\cos \phi_{i, \mu}^{\theta} \delta_{i+\hat{\mu}, j}+\left(\cos \phi_{i, \mu}^{\theta}+\cos \phi_{i-\hat{\mu}, \mu}^{\theta}\right) \delta_{i, j}-\cos \phi_{i-\hat{\mu}, \mu}^{\theta} \delta_{i-\hat{\mu}, j}\right), \\
& \left(M_{F P}^{s}\right)_{i, j}=\sum_{\mu}\left(-\sec ^{2} \frac{\phi_{i, \mu}^{\theta}}{2} \delta_{i+\hat{\mu}, j}+\left(\sec ^{2} \frac{\phi_{i, \mu}^{\theta}}{2}+\sec ^{2} \frac{\phi_{i-\hat{\mu}, \mu}^{\theta}}{2}\right) \delta_{i, j}-\sec ^{2} \frac{\phi_{i-\hat{\mu}, \mu}^{\theta}}{2} \delta_{i-\hat{\mu}, j}\right) .
\end{aligned}
$$

The boundary conditions are given by

$$
\theta_{i+N \hat{\mu}}=(-1)^{k} \theta_{i}, \quad \phi_{i+N \hat{\mu}, \mu}=(-1)^{k} \phi_{i, \mu},
$$

where $N$ is the total number of lattice sites in the $\mu$-direction. We have $k=0$ for periodic boundary conditions (PBC) and $k=1$ for anti-periodic boundary conditions (APBC). With $\mathrm{PBC}$ there is a global degree of freedom leading to a one-parameter family of solutions, as all the equations are unchanged under $\theta_{i} \rightarrow \theta_{i}+\vartheta, \forall i$ where $\vartheta$ is an arbitrary constant angle. We remove this degree of freedom by fixing one of the variables to be zero. In practice, we set $\theta_{(N, \ldots, N)}=0$.

From now on we concentrate only on these gauge-fixing functionals and let the $\left\{\phi_{i, \mu}\right\}$ take random values independent of the action. This corresponds to the strong coupling limit $\beta=0$. Note that this is sufficient to answer the question of whether or not $n[U]$ is orbit dependent, as every gauge orbit has a non-vanishing weight for any finite $\beta$.

It is worth noting that the functional (9) is identical to the hamiltonian of the random phase $\mathrm{XY}$ model, and that the functional for the trivial orbit is identical to the hamiltonian of the classical XY model in statistical physics [28, 29]. 


\subsection{Analytical results in 1 dimension}

Here we list the available analytical results in one dimension 2

1. For the naive functional with APBC [28, 14, the minima for any orbit are $\phi_{i}^{\theta}=0$ or $\pi$ for all $i=1, \ldots, N$, the number of minima for any orbit is 2 , and the number of stationary points is $2^{N}$.

2. For the naive functional with PBC [28, 29] the stationary points for any orbit and any odd number of sites $N$, are given by

$$
\begin{array}{rlrl}
\phi_{i}^{\theta} & =(-1)^{q_{i}} \phi_{N}^{\theta}+q_{i} \pi \quad \bmod 2 \pi, & & q_{i} \in\{0,1\}, \\
\phi_{N}^{\theta} & =\frac{N \bar{\phi}+2 \pi l-\pi \sum_{i=1}^{N-1} q_{i}}{1+\sum_{i=1}^{N-1}(-1)^{q_{i}}}, & l \in Z,
\end{array}
$$

with $\bar{\phi}:=\frac{1}{N} \sum_{i=1}^{N} \phi_{i}$. In fact, since we are interested in solutions of $\phi_{i}^{\theta}$ only modulo multiples of $2 \pi$, it is sufficient to consider

$$
l \in\left\{1, \ldots,\left|1+\sum_{i=1}^{N-1}(-1)^{q_{i}}\right|\right\}-\{0\} .
$$

The number of stationary points is $\sum_{j=0}^{N-1}|N-2 j|\left(\begin{array}{c}N-1 \\ j\end{array}\right)$, i.e., it increases exponentially with $N$. The minima occur when $q_{i}=0$, for all $i=1, \ldots, N-1$, and $\cos \phi_{N}^{\theta}>0$. Thus, the number of minima is bounded by $N$. The number of Gribov copies for the compact $U(1)$ theory in one dimension thus increases exponentially, but the number of Gribov copies in the first Gribov region increases only linearly. In [29], it was also shown that if $\bar{\phi}=\left(j+\frac{1}{2}\right) \pi$ with $j \in \mathbb{N}$, the FP operator is singular, i.e., for these orbits Gribov horizons do exist. $F$ evaluated at these stationary points is

$$
\left.F_{\phi}(\theta)\right|_{\theta=\theta_{0}}=N-\cos \phi_{N}^{\theta} \sum_{k=1}^{N}(-1)^{q_{k}} .
$$

The minima of this function are when both conditions (1) $q_{k}=0$ for all $k=1, \ldots, N-1$, and (2) $\cos \phi_{N}^{\theta}>0$ with $\phi_{N}^{\theta}$ given as Eqs. (16) and (17), are satisfied. Thus, the minima are given by

$$
\phi_{N}^{\theta}=\frac{\sum_{i=1}^{N} \phi_{i}+2 \pi l}{N}, \quad \text { with } \quad \phi_{i}^{\theta}=\phi_{N}^{\theta} \quad \bmod 2 \pi .
$$

It follows from (20) that function values at the minima accumulate near zero as $N \rightarrow \infty$. The proof of this is given in Appendix C]

3. We show in Appendix $\mathrm{A}$ that for the stereographic gauge with APBC, the FP operator is generically positive definite and hence the function $F_{\phi}^{s}(\theta)$ has only minima. In fact, there is only one minimum for any orbit in this case, $\phi_{i}^{\theta}=0 \bmod 2 \pi, i=1, \ldots, N$.

\footnotetext{
${ }^{2}$ We note that some analytical results for both $\mathrm{U}(1)$ and $\mathrm{SU}(2)$ in 1 dimension were already found in [47.
} 
4. For the stereographic gauge with $\mathrm{PBC}$, again, the FP operator is generically positive definite as shown in Appendix $\mathrm{A}$. There are $N$ minima for any orbit in this case 28, 48,

$$
\phi_{i}^{\theta}=\bar{\phi}-\frac{2 \pi}{N} r \bmod 2 \pi, \quad r=0, \ldots, N-1, \quad \bar{\phi}:=\frac{1}{N} \sum_{i=1}^{N} \phi_{i},
$$

i.e., the number of minima is orbit-independent and increases linearly with $N$.

\subsection{Some analytical results in 2 dimensions}

1. In any dimension, for the trivial orbit, there is only one global minimum with PBC, and two global minima with APBC, for both the naive and stereographic gauge.

Proof: Consider (9) defined on a lattice of size $N^{d}$. Let the components of $\theta$ be given by $\left\{\theta_{\left(i_{1}, \ldots, i_{d}\right)}\right\}$ where each $\theta_{\left(i_{1}, \ldots, i_{d}\right)} \in(-\pi, \pi], i_{1}, \ldots, i_{d}$ runs from $1, \ldots, N$, and $d$ is the dimension of the lattice. It is clear that the minimum value the naive Landau gauge-fixing functional, given in Eq.(9), can attain is 0. Since it is a non-negative function, when $F=0$, the only possible configuration is when each term in $F$ is zero, i.e.,

$$
\theta_{\left(i_{1}, \ldots, i_{d}\right)+\hat{\mu}}-\theta_{\left(i_{1}, \ldots, i_{d}\right)}=0 \quad \forall i_{1}, \ldots, i_{d} \in\{1, \ldots, N\}, \mu \in\{1, \ldots d\},
$$

which is the global minima. The stereographic Landau gauge-fixing functional is also a non-negative function and hence all the above arguments apply. Then any global minimum has to satisfy (22) and so with $\theta$ defined as above we must have $\theta_{\left(i_{1}, \ldots, i_{d}\right)+\hat{\mu}}=\theta_{\left(i_{1}, \ldots, i_{d}\right)}$ $\bmod 2 \pi$. Therefore, all $\theta_{\left(i_{1}, \ldots, i_{d}\right)}$ can be parameterised by only one of them, say, $\theta_{(1, \ldots, 1)}=$ $\theta_{\left(i_{1}, \ldots, i_{d}\right)+\hat{\mu}}$, for all $i_{1}, \ldots, i_{d}$ and $\mu$.

Now, for the APBC case, since $\theta_{(1, \ldots, 1, N+1)}=-\theta_{(1, \ldots, 1)}$ but also from the above arguments $\theta_{(1, \ldots, 1, N+1)}=\theta_{(1, \ldots, 1)} \bmod 2 \pi$. So we have $2 \theta_{(1, \ldots, 1)}=0 \bmod 2 \pi$. Thus either $\theta_{(1, \ldots, 1)}=0$ or $\theta_{(1, \ldots, 1)}=\pi$. So all global minima of (9) with APBC are lattices where all of the $\theta$-variables are zero or all $\theta$-variables are $\pi$. With PBC, we must fix one element on the lattice and require it to be zero. But as all elements are equal, this means that all the elements must be zero and thus the only global minimum of (9) with PBC is a lattice with all $\theta$-variables set to zero.

2. For a generic orbit for compact $\mathrm{U}(1)$, the number of stationary points for the naive functional is $\geq 2^{N}$ for APBC and $\geq 2^{N-1}$ for PBC. For the stereographic gauge, this lower bound is 1 . For generic $\mathrm{SU}\left(N_{c}\right)$ with $\mathrm{PBC}$, the number of stationary points is lowerbounded by $2^{\left(N_{c}-1\right)(N-1)}$ (See Appendix $\mathrm{B}$ for a proof).

3. If $\theta$ is a minimum of the naive or stereographic lattice Landau gauge with APBC, then one can construct another minimum by adding $\pi$ to every $\theta_{i}$.

This is easy to see in one dimension: let $\theta_{i} \rightarrow \theta_{i}+\pi$, for $i=1, \ldots, N-1$, then $\phi_{i}^{\theta} \rightarrow \phi_{i}^{\theta}$. For $i=N$, this transformation leaves $\phi_{N}^{\theta}=\phi_{N}-\theta_{1}-\theta_{N} \rightarrow \phi_{N}-\theta_{1}-\theta_{N}-2 \pi=\phi_{N}^{\theta} \bmod 2 \pi$. Due to the periodicity of the trigonometric functions there is no effect of this transformation in either the gauge-fixing equations themselves or in the hessian. It is straightforward to extend the same argument to any dimension. 
4. For the trivial orbit in $d$ dimensions, for a symmetric $N^{d}$ lattice, if $\theta=\left\{\theta_{i_{1}, \ldots, i_{d}}\right\}$ is a minimum, then $\tilde{\theta}= \pm G \theta$ is also a minimum, where $G$ is any lattice symmetry operation, including lattice translations, rotations and reflections (or axis permutations). This follows straightforwardly from the symmetries of the defining equations. Specifically, for example in two dimensions, if $\theta=\left\{\theta_{i j}\right\}$ is a minimum of the naive or stereographic gauge fixing functional, then $\theta^{T}=\left\{\theta_{j i}\right\}$ is also a minimum, as the following argument shows.

Let $\theta$ be a solution of Eq.(9), i.e., it satisfies the following equations for all $i, j=1, \ldots, N$,

$$
\begin{aligned}
\frac{\partial F}{\partial \theta_{i, j}}= & \sin \left(\theta_{i, j}-\theta_{i-1, j}\right)-\sin \left(\theta_{i+1, j}-\theta_{i, j}\right) \\
& +\sin \left(\theta_{i, j}-\theta_{i, j-1}\right)-\sin \left(\theta_{i, j+1}-\theta_{i, j}\right) \\
\text { (relabel } i \rightarrow j, j \rightarrow i)= & \sin \left(\theta_{j, i}-\theta_{j-1, i}\right)-\sin \left(\theta_{j+1, i}-\theta_{j, i}\right) \\
& +\sin \left(\theta_{j, i}-\theta_{j, i-1}\right)-\sin \left(\theta_{j, i+1}-\theta_{j, i}\right) \\
= & -\frac{\partial F}{\partial \theta_{j, i}}=0 .
\end{aligned}
$$

Thus, $\theta^{T}$ is a stationary point of (9). To prove that $\theta^{T}$ is a minimum, we note that the Hessian (13) for the naive and similarly for the stereographic gauge in the case of the trivial orbit are both symmetric under the interchange of coordinates, and therefore the hessian evaluated at $\theta$ has the same eigenvalues as the hessian evaluated at $\theta^{T}$. Thus, if $\theta$ is a minimum, then $\theta^{T}$ is also a minimum.

It is easy to see that this permutation symmetry holds for any dimension, and works for both naive and stereographic gauge fixing functionals and for stationary points in general, for the trivial orbit. Since there are $4 N^{d} d$ ! elements of the symmetry group for a $N^{d}$ lattice ( $N^{d}$ lattice translations, $d$ ! permutations, plus reflection and sign change), this means that if $\theta$ is a stationary point of a $d$-dimensional functional then the function value at $\theta$ will be $4 N^{d} d$ !-fold degenerate unless $\theta$ maps onto itself under a subset of the symmetry operations. For example, in $d=2$ the only permutation of spatial coordinates is a transposition, giving a degeneracy of $8 N^{2}$, but is reduced by a factor two for any skew-symmetric stationary point, $\theta^{T}=-\theta$. The function value of zero is non-degenerate, as $\theta=0$ maps onto itself under all symmetry operations.

If we choose to impose periodic boundary conditions, then we essentially choose the fixed site to have lattice coordinate in our case $(N, \ldots, N)$, which is fixed under any permutation and sign flip, but not under lattice rotations or reflections. This means that the generic degeneracy is reduced to $2 d$ !.

\section{Numerical polynomial homotopy continuation method}

In general, systems of non-linear equations are extremely difficult to solve. However, if the non-linearity in the system is polynomial-like, then the situation is enhanced due to the recently developed algebraic geometry methods. In particular, we will use the so-called numerical polynomial homotopy continuation (NPHC) method [49] to find all the solutions of the gauge-fixing equations. This method was introduced in particle physics and statistical mechanics first in [28] 
and applied in 50, 51, 52, 53, 54, 55, 56, 57, 58. Below, first we show that the problem of solving the extremising equations in terms of the $\theta$-variables can be transformed into that of solving a system of multivariate polynomial equations. Then we describe the numerical polynomial homotopy continuation method, which can be used to find all the solutions of a given system of polynomial equations numerically. Finally, we give our results for the problem at hand.

To convert the naive gauge-fixing equations, we can first use trigonometric identities to rewrite (11) as

$$
\begin{aligned}
f_{i}(c, s)=\sum_{\mu}\left(c_{i}\left(c_{i+\hat{\mu}} \sin \phi_{i, \mu}-c_{i-\hat{\mu}} \sin \phi_{i-\hat{\mu}, \mu}+s_{i-\hat{\mu}} \cos \phi_{i-\hat{\mu}, \mu}+s_{i+\hat{\mu}} \cos \phi_{i, \mu}\right)\right. \\
\left.\quad+s_{i}\left(s_{i+\hat{\mu}} \sin \phi_{i, \mu}-s_{i-\hat{\mu}} \sin \phi_{i-\hat{\mu}, \mu}-c_{i+\hat{\mu}} \cos \phi_{i, \mu}-c_{i-\hat{\mu}} \cos \phi_{i-\hat{\mu}, \mu}\right)\right),
\end{aligned}
$$

where we have written $s_{i}:=\sin \theta_{i}$ and $c_{i}:=\cos \theta_{i}$. This is merely a change of notation. However, we can now add additional equations to the system for each site $i$, namely,

$$
g_{i}(c, s)=s_{i}^{2}+c_{i}^{2}-1=0,
$$

The combined system of all $f_{i}(c, s)$ and $g_{i}(c, s)$ is not just a change of notation: all the $c_{i}$ and $s_{i}$ are now algebraic variables and the equations are multivariate polynomial equations, i.e., the fact that $c_{i}$ and $s_{i}$ are originally $\sin \theta_{i}$ and $\cos \theta_{i}$ is taken care of by the constraint equations (26). In general, for a lattice with $N$ lattice sites, we have in total $2 N$ polynomial equations and $2 N$ variables.

To convert the gauge-fixing equations arising for the stereographic gauge, we first simply expand (12) using the trigonometric identity

$$
\tan \frac{x+y+z}{2}=\frac{\sin x+\cos z \sin y+\cos y \sin z}{\cos x+\cos y \cos z-\sin y \sin z},
$$

to obtain

$$
f_{i}^{s}(c, s)=\sum_{\mu}\left(\frac{\sin \phi_{i, \mu} c_{i}-\cos \phi_{i, \mu} s_{i}+s_{i+\hat{\mu}}}{\sin \phi_{i, \mu} s_{i}+\cos \phi_{i, \mu} c_{i}+c_{i+\hat{\mu}}}-\frac{\sin \phi_{i-\hat{\mu}, \mu} c_{i-\hat{\mu}}-\cos \phi_{i-\hat{\mu}, \mu} s_{i-\hat{\mu}}+s_{i}}{\sin \phi_{i-\hat{\mu}, \mu} s_{i-\hat{\mu}}+\cos \phi_{i-\hat{\mu}, \mu} c_{i-\hat{\mu}}+c_{i}}\right),
$$

which can again be translated into the same polynomial form as above. Here, the difference is that the above equations are not in the 'polynomial form' due to the denominator. We can clear the denominators out by multiplying them with the numerators appropriately and assuming that none of the denominators are zero (such solutions can be sorted and thrown out once all the solutions are obtained).

For example, for the trivial orbit on a one-dimensional lattice with $N=3$ and APBC, Eq. (27) simplifies to

$$
\frac{s_{2}-s_{1}}{c_{1}+c_{2}}-\frac{s_{1}+s_{3}}{c_{1}+c_{3}}=\frac{s_{3}-s_{2}}{c_{2}+c_{3}}-\frac{s_{2}-s_{1}}{c_{1}+c_{2}}=\frac{-s_{1}-s_{3}}{c_{1}+c_{3}}-\frac{s_{3}-s_{2}}{c_{2}+c_{3}}=0 .
$$

After clearing out the denominators, the equations become

$$
\begin{aligned}
-2 c_{1} s_{1}-c_{2} s_{1}-c_{3} s_{1}+c_{1} s_{2}+c_{3} s_{2}-c_{1} s_{3}-c_{2} s_{3} & =0 \\
c_{2} s_{1}+c_{3} s_{1}-c_{1} s_{2}-2 c_{2} s_{2}-c_{3} s_{2}+c_{1} s_{3}+c_{2} s_{3} & =0, \\
-c_{2} s_{1}-c_{3} s_{1}+c_{1} s_{2}+c_{3} s_{2}-c_{1} s_{3}-c_{2} s_{3}-2 c_{3} s_{3} & =0 \\
1-y\left(c_{1}+c_{2}\right)\left(c_{1}+c_{3}\right)\left(c_{2}+c_{3}\right) & =0,
\end{aligned}
$$


where the last equation is added to ensure that the denominators are never zero, and $y$ is an additional variable. Thus, the final set of the equations is in polynomial form. One can then solve this system using the NPHC method.

\subsection{The method}

Let us consider a system of multivariate polynomial equations, say $P(x)=0$, where $P(x)=$ $\left(p_{1}(x), \ldots, p_{m}(x)\right)^{T}$ and $x=\left(x_{1}, \ldots, x_{m}\right)^{T}$, which is known to have isolated solutions, e.g., the above mentioned gauge fixing equations after eliminating the global gauge freedom. Now, the Classical Bezout Theorem asserts that for a system of $m$ polynomial equations in $m$ variables, for generic values of coefficients, the maximum number of solutions in $\mathbb{C}^{m}$ is $\prod_{i=1}^{m} d_{i}$, where $d_{i}$ is the degree of the $i$ th polynomial. This bound, the classical Bezout bound (CBB), is exact for generic values (i.e., roughly speaking, non-zero random values) of coefficients, e.g., for the onedimensional naive (or minimal) gauge fixing equations with $N$ of lattice sites and with APBC, this number is $2^{2 N}$ (because there are $2 N$ polynomials each of which is a degree 2 polynomial). The genericity is well-defined and the interested reader is referred to Ref. [49, 59] for details.

Based on the CBB, a homotopy can be constructed as

$$
H(x, t)=\gamma(1-t) Q(x)+t P(x),
$$

where $\gamma$ is a random complex number. $Q(x)=\left(q_{1}(x), \ldots, q_{m}(x)\right)^{T}$ is a system of polynomial equations with the following properties:

1. the solutions of $Q(x)=H(x, 0)=0$ are known or can be easily obtained. $Q(x)$ is called the start system and the solutions are called the start solutions,

2. the number of solutions of $Q(x)=H(x, 0)=0$ is equal to the CBB for $P(x)=0$,

3. the solution set of $H(x, t)=0$ for $0 \leq t \leq 1$ consists of a finite number of smooth paths, called homotopy paths, each parameterised by $t \in[0,1)$, and

4. every isolated solution of $H(x, 1)=P(x)=0$ can be reached by some path originating at a solution of $H(x, 0)=Q(x)=0$.

The start system $Q(x)=0$ can for example be taken to be

$$
Q(x)=\left(\begin{array}{c}
x_{1}^{d_{1}}-1 \\
\vdots \\
x_{m}^{d_{m}}-1
\end{array}\right)=0,
$$

where $d_{i}$ is the degree of the $i^{t h}$ polynomial of the original system $P(x)=0$. Eq. (31) is easy to solve and guarantees that the total number of start solutions is $\prod_{i=1}^{m} d_{i}$, all of which are non-singular.

One can then track all the paths corresponding to each solution of $Q(x)=0$ from $t=0$ to $t=1$ and reach $P(x)=0=H(x, 1)$. By implementing an efficient path tracker algorithm, e.g., Euler predictor and Newton corrector methods, all isolated solutions of a system of multivariate 
polynomials system can be obtained. The complex random number $\gamma$ is crucial here: it has been shown [49] that for a randomly chosen $\gamma$, there are no singularities (i.e., paths do not cross each other) for $t \in[0,1)$. This ensures that in the end we get all the solutions. In this respect, the NPHC method has a great advantage over all other known methods for finding stationary points or minima.

There are several sophisticated numerical packages well-equipped with path trackers such as Bertini [60, PHCpack 61, PHoM [62] and HOM4PS2 [63, 59]. They all are available as freewares from the respective research groups. We mainly use Bertini, HOM4PS2 and PHCpack to get the results in this paper: for each of the systems, we use at least two of the packages to cross-check the results.

\subsection{Results}

Before proceeding to the results, it should be noted that a solution here means a set of values of $s_{i}$ and $c_{i}$ (for the naive or minimal gauge) or $t_{i}$ 's (for the stereographic gauge) satisfies all of the equations with tolerances $10^{-10}$. All the solutions come with real and imaginary parts. A solution is a real solution if the imaginary part of each of the variables is less than or equal to the tolerance $10^{-6}$ (below which the number of real solutions does not change, i.e., it is robust for all the cases we consider in this discussion). The original trigonometric equations are satisfied with tolerance $10^{-10}$ after the $s_{i}$ and $c_{i}$ (or $t_{i}$ ) are transformed back to $\theta_{i}$. All these solutions can be further refined to an arbitrary precision.

We present the results for the two-dimensional naive functional by classifying the obtained solutions in terms of the number of positive and negative eigenvalues of the corresponding Hessian matrix, or the FP operator, because then we can use the Neuberger zero as a necessary condition for having all the solutions. Before proceeding to the two-dimensional case, we note that we have reproduced the known analytical results in the one-dimensional case for both APBC and PBC naive and stereographic gauge cases using the NPHC method, up to $N=25$ for various random orbits.

We now explore the simplest non-trivial case in higher dimensional lattices which is the naive functional on a $3 \times 3$ lattice with the trivial orbit (TO) and 10 random orbits (ROs) (i.e., randomly chosen $\left.\phi_{i, \mu} \in(-\pi, \pi]\right)$ with $\mathrm{PBC}$ and APBC. For these cases, the CBB values are 65536 and 262144, respectively.

The results of our runs are summarised in Tables 1 and 2 for APBC and PBC, respectively. We find that:

1. For the trivial orbit with APBC, there are a total of 3768 real solutions, i.e., Gribov copies. Out of these, there are 1952 solutions which have zero FP determinant with tolerance $10^{-8}$. These singular solutions lie exactly on the Gribov horizon. They can be further classified in terms of the number of zero eigenvalues of the FP operator at each of the solutions. This amounts to classifying the singular solutions of the polynomial system in terms of their multiplicities using the so-called deflation singularities technique [64. The remaining 1816 real solutions have non-singular $M_{F P}$. Similarly, the trivial orbit with PBC has 30 singular (Gribov horizon) solutions out of a total of 1112 Gribov copies.

2. There are no singular solutions for any of the random orbits for either PBC or APBC. The total number of Gribov copies for each random orbit fluctuates around $\sim 2500$ for the 


\begin{tabular}{|c|c|c|c|c|c|c|c|c|c|c|c|c|}
\hline & & & \multicolumn{10}{|c|}{ of negative eigenvalues } \\
Orbit & $N_{\text {tot }}$ & $N_{n s}$ & 0 & 1 & 2 & 3 & 4 & 5 & 6 & 7 & 8 & 9 \\
\hline \hline TO & 3768 & 1816 & 2 & 18 & 216 & 342 & 330 & 330 & 342 & 216 & 18 & 2 \\
\hline RO1 & 2480 & 2480 & 2 & 58 & 202 & 402 & 576 & 576 & 402 & 202 & 58 & 2 \\
\hline RO2 & 2304 & 2304 & 10 & 36 & 148 & 382 & 576 & 576 & 382 & 148 & 36 & 10 \\
\hline RO3 & 2440 & 2440 & 12 & 66 & 196 & 374 & 572 & 572 & 374 & 196 & 66 & 12 \\
\hline RO4 & 2584 & 2584 & 10 & 54 & 210 & 444 & 574 & 574 & 444 & 210 & 54 & 10 \\
\hline RO5 & 2408 & 2408 & 2 & 44 & 202 & 404 & 552 & 552 & 404 & 202 & 44 & 2 \\
\hline RO6 & 2672 & 2672 & 10 & 60 & 208 & 460 & 598 & 598 & 460 & 208 & 60 & 10 \\
\hline RO7 & 2504 & 2504 & 8 & 46 & 190 & 426 & 582 & 582 & 426 & 190 & 46 & 8 \\
\hline RO8 & 2304 & 2304 & 6 & 48 & 152 & 362 & 584 & 584 & 362 & 152 & 48 & 6 \\
\hline RO9 & 2352 & 2352 & 6 & 50 & 182 & 384 & 554 & 554 & 384 & 182 & 50 & 6 \\
\hline RO10 & 2382 & 2382 & 6 & 44 & 180 & 402 & 558 & 558 & 402 & 180 & 44 & 6 \\
\hline
\end{tabular}

Table 1: Summary of the Gribov copies of the naive lattice Landau gauge for $3 \times 3$ lattice, for different orbits with APBC. $N_{\text {tot }}$ is the total number of real solutions and $N_{n s}$ the number of non-singular solutions. RO denotes random orbit while TO denotes the trivial orbit. The number of global minima is 2 for all orbits.

\begin{tabular}{|c|c|c|c|c|c|c|c|c|c|c|c|}
\hline & & & \multicolumn{9}{|c|}{ No of negative evalues } \\
Orbit & $N_{\text {tot }}$ & $N_{n s}$ & 0 & 1 & 2 & 3 & 4 & 5 & 6 & 7 & 8 \\
\hline \hline TO & 1112 & 966 & 1 & 9 & 76 & 174 & 117 & 159 & 234 & 156 & 40 \\
\hline TO mag & 1034 & 1034 & 1 & 17 & 110 & 188 & 173 & 156 & 193 & 156 & 40 \\
\hline RO1 & 480 & 480 & 3 & 15 & 50 & 95 & 121 & 116 & 65 & 14 & 1 \\
\hline RO2 & 476 & 476 & 1 & 12 & 45 & 99 & 129 & 109 & 62 & 18 & 1 \\
\hline RO3 & 498 & 498 & 2 & 15 & 54 & 100 & 132 & 115 & 59 & 19 & 2 \\
\hline RO4 & 542 & 542 & 1 & 16 & 56 & 108 & 149 & 131 & 64 & 16 & 1 \\
\hline RO5 & 470 & 470 & 1 & 9 & 46 & 105 & 138 & 110 & 49 & 11 & 1 \\
\hline RO6 & 494 & 494 & 1 & 21 & 68 & 111 & 120 & 102 & 56 & 13 & 2 \\
\hline RO7 & 506 & 506 & 2 & 16 & 62 & 130 & 138 & 89 & 49 & 18 & 2 \\
\hline RO8 & 484 & 484 & 3 & 18 & 43 & 101 & 142 & 104 & 52 & 19 & 2 \\
\hline RO9 & 484 & 484 & 2 & 9 & 49 & 114 & 145 & 104 & 44 & 15 & 2 \\
\hline RO10 & 466 & 466 & 1 & 13 & 47 & 97 & 128 & 104 & 55 & 19 & 2 \\
\hline
\end{tabular}

Table 2: As Table 1 but for PBC. TO mag field denotes the trivial orbit with the modified functional (32) with $h=0.01$ (see main text for explanation). The number of global minima is 1 for all orbits. 
APBC case and around $\sim 500$ for the PBC case. We see that the total number of Gribov copies is an orbit-dependent quantity in both cases.

3. All the Gribov copies (excluding the Gribov horizons) can be classified by the number of negative eigenvalues, as shown in Tables 1 and 2, In the APBC case, one can see a perfect symmetry among the Gribov copies in the number of solutions classified according to the negative eigenvalues of the FP operators evaluated at these solutions. This symmetry yields a perfect cancellation of the signs of the FP determinants, giving rise to the Neuberger zero. For the PBC case, there is no such manifest symmetry. However, we can easily check that the sum of signs of the FP determinants is still zero for all orbits, even though the total number of Gribov copies is an orbit-dependent quantity.

4. The solutions with no negative eigenvalues are the minima. We see that for both types of boundary conditions, the total number of (local and global) minima is orbit-dependent. The global minima can be determined by computing the functional $F$ with appropriate boundary conditions for each of these minima and identifying those giving the lowest value for $F$. We find that the number of global minima is orbit-independent. It should be emphasised that though there are two global minima for the APBC case, both of them are trivial copies of each other.

5. Strictly speaking, because there are singular solutions for the trivial orbit with both APBC and PBC, Morse theory does not directly apply here 3 Hence one should not expect that the sum of the signs of the FP determinants would give the Euler characteristic in such a case, and this can indeed be seen for the trivial orbit with PBC in Table2. Instead, we have to work with Morse-Bott theory, a discussion of which is beyond the scope of this article. Note that Schaden's equivariant construction takes this fact into account. However, a conventional way to remove such singular solutions is to add an external magnetic field term in the original height function, which in our case amounts to adding an external magnetic field term to Eq. (9), i.e.,

$$
F_{\phi}(\theta) \rightarrow F_{\phi}^{m}(\theta)=F_{\phi}(\theta)+h \sum_{i=1}^{N} \cos \theta_{i} .
$$

The new system of equations can again be translated to polynomial form and solved using the NPHC method. The results for the trivial orbit with PBC (taking $h=0.01$ ) are also reported in Table 2, where one can again see that the sum of the signs of the FP determinants is zero.

From our results we can also find the distribution of functional values $F$, broken down by the number of negative eigenvalues (ie, for each Gribov region). The results are shown in Fig. 1 for antiperiodic boundary conditions; the results for periodic boundary conditions are similar. We see that the functional values cluster around different values for different $n$, with a peak of

\footnotetext{
${ }^{3}$ It is important to mention that we have observed using the numerical algebraic geometry methods that there is a positive dimensional real component of dimension at least one in the solution space of $3 \times 3$ lattices. This is surprising because after eliminating the global gauge degree of freedom, there should only be isolated solutions left. Further studying this phenomenon and whether this is of any physical significance is very interesting but beyond the scope of the present article.
} 


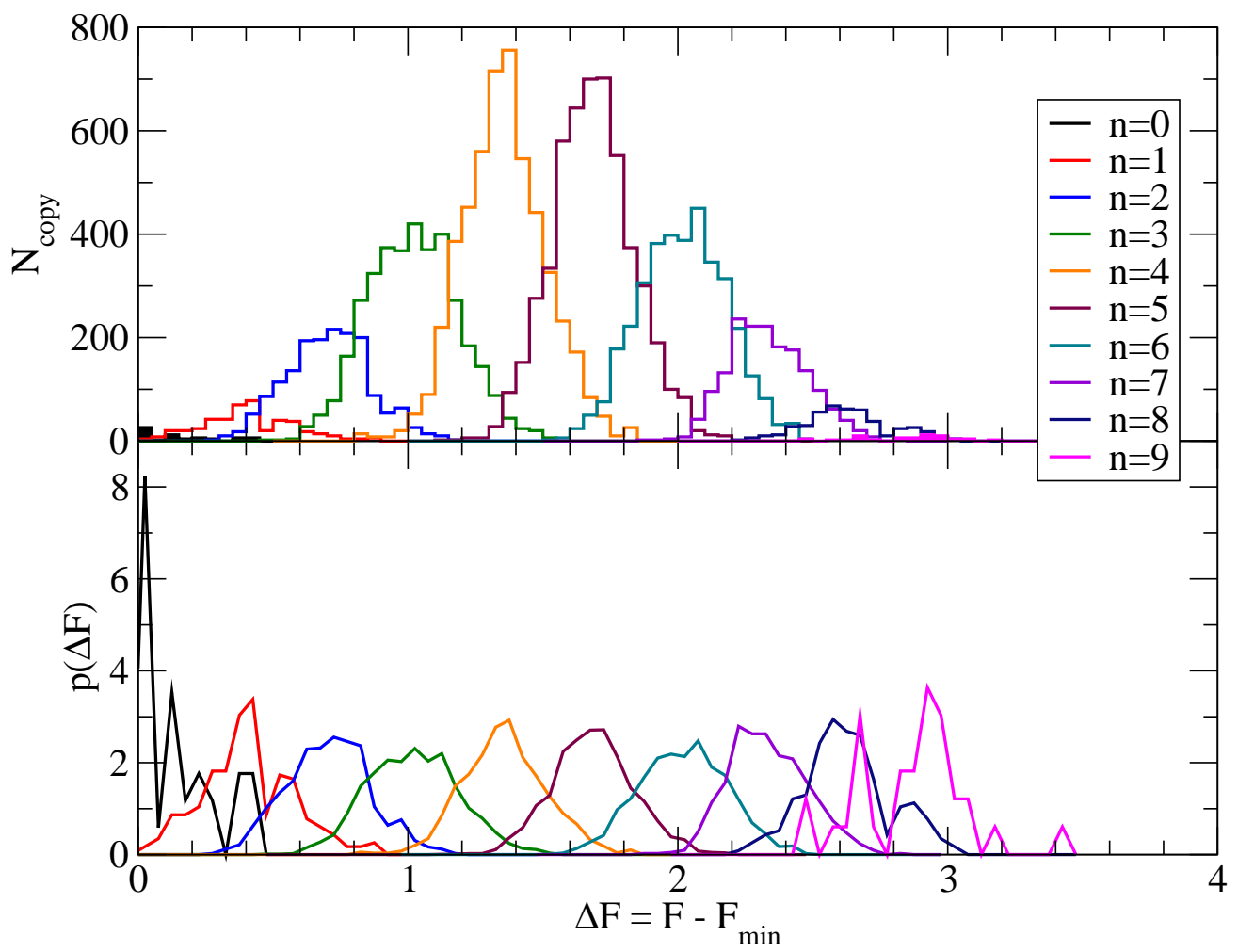

Figure 1: Distribution of the values of the gauge fixing functional relative to the absolute minimum for each orbit, $\Delta F=F-F_{\min }$, for 10 random orbits in the naive gauge on the $3 \times 3$ lattice with antiperiodic boundary conditions, and for different number $n$ of negative eigenvalues. Top: Histogram of the absolute number $N_{\text {copy }}$ of copies found in each bin; Bottom: The distribution separately normalised for each value of $n$.

the distribution which is roughly linear in $n$. However, there is a substantial overlap between the distributions for different $n$, and in particular (because of the much larger number of saddle points than of minima) there are many saddle points with the same functional value as that of typical minima.

It would clearly be interesting to find out how this pattern changes for larger lattice sizes, but this is beyond the scope of this study.

The equations for the stereographic gauge are quite difficult to solve: the polynomial form of the equations is both dense and of high degree. We have only been able to find all the solutions for the trivial orbit on the $3 \times 3$ lattice with periodic boundary conditions, where the number of Gribov copies was found to be 5256. This is much larger than the 112 copies found in the naive gauge, showing that the exponential suppression in 1 dimension does not carry over to higher dimensions. 


\section{Numerical minimisation}

So far we have collected or worked out some analytical results for solutions in the one dimensional lattices and have used the NPHC method to find all the solutions for the $3 \times 3$ lattices. For lattices larger than $3 \times 3$, we have not yet been able to obtain results using the NPHC method. To get results for the bigger lattices, we have to rely on the traditional 'brute force' methods. Note that for these traditional methods, there is no guarantee of finding all solutions nor all minima of a given function. But our strategy is to first study the cases for which we already know all the solutions: we reproduce the results for the one dimensional lattices and for the $3 \times 3$ lattice using the traditional method. This gives us a hint on how much computational effort we will require for larger lattices.

In particular, we have used the conjugate gradient algorithm 65] to numerically obtain minima and stationary points for both the naive and stereographic gauge. Once the conjugate gradient algorithm has converged we check that $\left\|\nabla_{\theta} F\right\|^{2} \leq \epsilon_{1}$ where $\epsilon_{1}$ is sufficiently small to ensure that we have actually obtained a minimum. We reduce $\theta \bmod 2 \pi$ to make sure the values are in the interval $(-\pi, \pi]$. We have to set a tolerance, $\epsilon_{2}$, on how close $\theta_{i}$ has to be to $\pi$ or 0 to be considered either, respectively, and apply these changes. Errors occurred when trying to determine the total number of minima if this flag was not in place. Also, if $\left|\theta_{i}+\pi\right| \leq \epsilon_{2}$ then $\theta_{i} \rightarrow \pi$, since $\theta_{i} \in(-\pi, \pi]$, not $[-\pi, \pi]$.

To obtain "all" minima, we generate a series of random initial guesses for the variables $\theta_{i}$, and successively minimise the given functional from each initial guess. We can then count the number of unique minima. After increasing the number of samples sufficiently, it is noted that in most cases the total number of unique minima that the function converges to stops increasing and thus we can say to have completely sampled the solution space of the function. Examples of this are shown in Fig. 2, We note that the number of unique minima found is usually far smaller than the number of samples used. We call this method the Monte Carlo Conjugate Gradient Method (MCCGM).

\subsection{1 dimension}

Since our method does not guarantee that we find all the minima, we first show that we reproduce all the minima in the one-dimensional case where rigorous analytical results are available. Indeed, we have reproduced all the minima for lattices up to $N=1000$, for both the naive/minimal and stereographic gauge with APBC. We also reproduced all the minima with PBC for up to $N=16$ for the naive functional and up to $N=7$ for stereographic gauge. In Figure 3 , we plot the number of minima as a function of the number of lattice sites for both gauges with both types of boundary conditions With APBC the number of minima is always 2 for both the stereographic and naive gauge functional. With $\mathrm{PBC}$, the number of minima is orbit-dependent, and correspondingly two possible values are shown for each $N$. These results are in complete agreement with the analytical results found previously.

To separate out the global minimum, we have then evaluated the corresponding functional value at each of the local minima, and identified the minimum (or minima) with the lowest functional value. To remove any uncertainty, we have also used Matlab's in-built routines for Simulated Annealing and Genetic Algorithm to find the global minimum for the one-dimensional cases, and these global minima always match the one obtained from the conjugate gradient minimisation. 


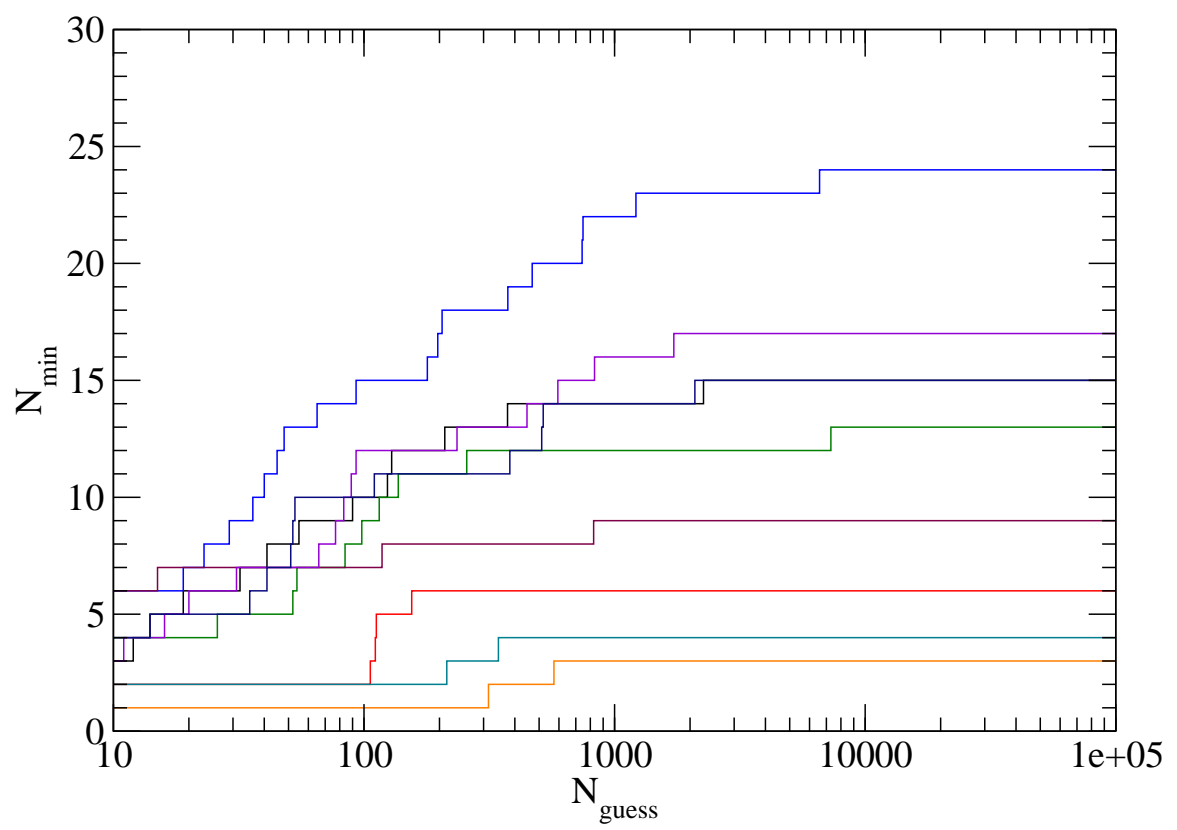

Figure 2: Number of distinct minima $N_{\min }$ found as a function of the number of random initial guesses $N_{\text {guess }}$ (start vectors for the CG minimisation), for 9 different random orbits in the naive gauge on the $5 \times 5$ lattice with periodic boundary conditions. Note the logarithmic scale on the horizontal axis.

The global minima from all these methods match up to $N=35$ for the naive APBC case. For lattice sizes larger than this the Matlab minimisation routines became inefficient both in terms of memory and computation time. The MCCGM was found to be superior to both of these methods as it took less time and memory to find all minima compared to the Simulated Annealing and Genetic Algorithm routines. We find that for all the random orbits, the number of global minima is the same (one for PBC and two for APBC).

In figure 4 we show the distribution of function values at each minimum for 100 different orbits, for the one-dimensional naive (minimal) gauge with $\mathrm{PBC}$, for four different values of the lattice size $N$. We see that for small $N$ the function values are almost uniformly distributed between 0 and 1 , but as $N$ grows the function values tend to accumulate near 0 . This is in accordance with the arguments in Appendix $[$ that function values accumulate near zero as $N \rightarrow \infty$.

For the stereographic gauge, our numerical minimisation approach broke down beyond $N=16$ for $\mathrm{PBC}$, in that we failed to find all the minima even when increasing the number of initial guesses to beyond $10^{7}$ (for APBC it worked fine up to $N=1000$ ). In this case each minimum is located in infinitely deep potential wells, and which particular minimum we find is uniquely determined by the initial guess. A strategy to select the initial guesses by uniformly sampling these potential wells in the PBC case has proved elusive. The same problem affects the 2dimensional stereographic gauge even more strongly, as we shall see in the next section. 

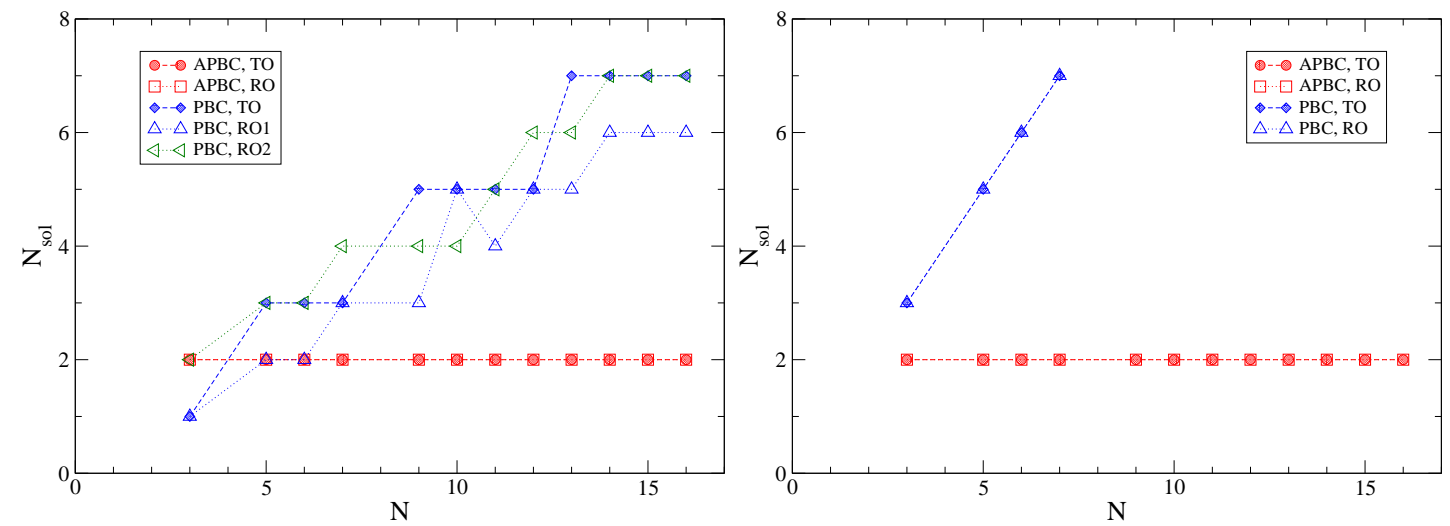

Figure 3: The number of (local and global) minima $N_{\min }$ vs the number of lattice sites $N$, for naive (left) and stereographic gauge (right) in 1 dimension, with PBC and APBC, for the trivial orbit (TO) and random orbits (RO).

\subsection{2 dimensions}

\subsubsection{Naive lattice Landau gauge}

Table 3 and Fig. 5 show the number of minima $N_{\min }$ for the trivial orbit of the naive functional with both boundary conditions. Because of the permutation symmetries noted in Sec. 3.2, each minimum will in general be $n$-fold degenerate, where $n=16 N^{2}$ for APBC and $n=4$ for PBC. For smaller lattice sizes in particular, some of the minima are invariant under a subset of these permutations, reducing the level of degeneracy. For periodic boundary conditions, we find a higher level of degeneracy than naively expected, suggesting additional symmetries. Indeed, studying the individual solutions we find that they consist of permutations of the same numbers. We have been able to classify some of these permutations, but have not as yet found any systematic pattern that is valid for generic lattice sizes.

It is clear that the number of distinct minima increases considerably for $N>7$, but it is not possible to conclude from our results on these small lattices whether it increases polynomially or exponentially with $N$.

In Figure 6 we plot the function values at each minimum against $N$ for each lattice of size $N \times N$ up to a lattice of size $12 \times 12$. This shows that the function values of 2-D minima of the trivial orbit appear to decay towards zero, just as in the 1-D case. This is again in accordance with the arguments in Appendix C For the lowest non-trivial minimum, which clearly can be seen to decrease with $N$, we see by inspection that this consists of a layering of 1-dimensional minima, as described in Appendix [C, whose function values (at least for the lower-lying ones) can be shown to decrease with $N$. However, we do not have a proof that most minima approach a function value of zero. We shall see later that this behaviour does not carry over to random orbits in 2 dimensions.

To study the random orbit case, we have generated $N_{\text {orb }}$ random orbits for each lattice size. For each orbit, we have found all the solutions, including the global minimum. The details are given 


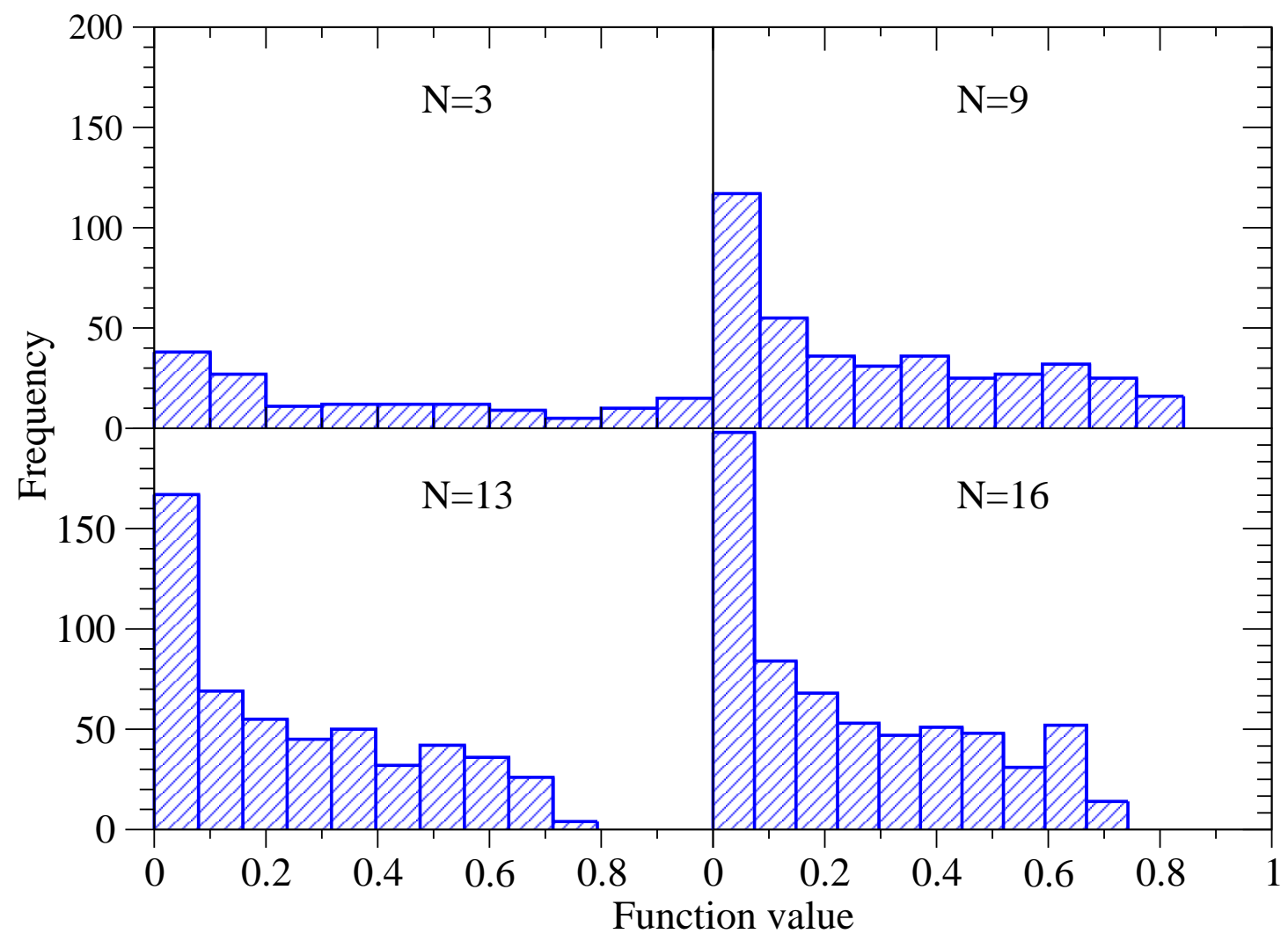

Figure 4: Histogram of function values at each minimum for 100 different orbits, for the onedimensional naive functional with $\mathrm{PBC}$.

in Table 4.

Our first observation concerns the number of solutions (Gribov copies), which, as expected, is orbit-dependent and increases with $N$. Unlike the trivial orbit case, all the minima are distinct for the random orbits (or twofold degenerate for antiperiodic boundary conditions). The number of minima for the $3 \times 3$ lattice agrees with that found using the NPHC method in section 4 Note that because of the twofold degeneracy for APBC we have divided the total number of solutions by 2 to factor this out. As we can see in Fig. 7 the number of solutions increases exponentially with $N$, with roughly the same rate of increase for periodic and antiperiodic boundary conditions. We also found that there is always 1 global minimum for $\mathrm{PBC}$ and 2 degenerate global minima for APBC. For a more detailed view, figure 8 shows histograms of the number of solutions for the four smallest lattice sizes. We do not observe any significant change in the shape of the distribution as $N$ increases, at least for these small lattices.

We now turn to the values of the gauge fixing functional $F$ for the different lattice sizes. Two issues are of particular interest: firstly, how does the absolute minimum change as a function of $N$; and secondly, how does the spread of function values change as $N$ changes? If the first 


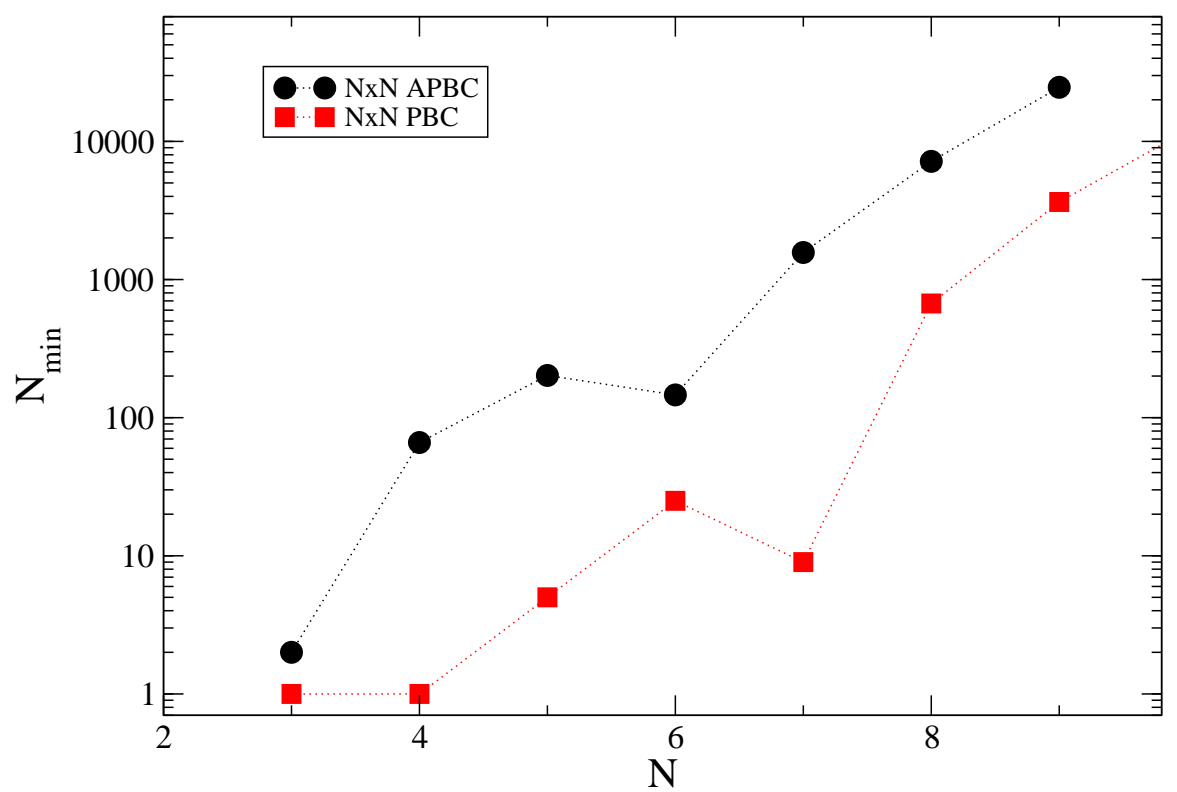

Figure 5: Plot of the lattice size $N$ vs number of (global and local) minima of the naive functional in 2D, with $\mathrm{PBC}$ and $\mathrm{APBC}$, for the trivial orbit.

Gribov region becomes equivalent to the fundamental modular region in the large volume limit, as conjectured, then we might expect the functional values to accumulate closer to the absolute minimum for larger volumes. We therefore determine the absolute minimum as well as the median value of the functional at the minimum for each random orbit. The results are shown in Table 4

Unlike the case for the trivial orbit, we find no evidence that the absolute minimum $F_{\min }$ decreases towards zero as $N$ is increased. Instead, $F_{\min }$ appears to converge to a value around 0.4. This is not unsurprising, as we are working with random gauge configurations (corresponding to the strong coupling limit), which are not smooth and should therefore not be expected to yield smooth gauge fixed configurations characterised by a small value for the gauge fixing functional.

We also find no clear evidence that the typical (or median) functional value at the minima approaches the absolute minimum as $N$ increases. If anything, the indication is that the opposite is the case for periodic boundary conditions, while for antiperiodic boundary conditions the median value may be getting closer to the absolute minimum. This is borne out by the distribution of functional values relative to the absolute minimum, shown in Fig. 9. For both boundary conditions the distribution goes from being fairly flat to more sharply peaked as $N$ increases, but for APBC the peak appears to shift towards zero, while for PBC the peak position stays constant or increases slightly.

As an aside, we observe that the lower minima are always the more likely to be found by the minimisation algorithm, with the global minimum among the first to be found. If instead of counting each Gribov copy equally, we weight them with how frequently they are found by the 


\begin{tabular}{|l|rrrrrrrr|}
\hline Lattice Size $N$ & 3 & 4 & 5 & 6 & 7 & 8 & 9 & 10 \\
\hline$N_{\text {min }}(\mathrm{APBC})$ & 2 & 66 & 202 & 146 & 1570 & 7170 & 24626 & \\
$\left(N_{\min }-2\right) / N^{2}$ & 0 & 4 & 8 & 4 & 32 & 112 & 304 & \\
$N_{\text {distinct }}$ & 1 & 2 & 2 & 2 & 4 & 13 & 20 & \\
$N_{\text {min }}(\mathrm{PBC})$ & 1 & 1 & 5 & 25 & 9 & 671 & $\gtrsim 4400$ & $\gtrsim 12000$ \\
$N_{\text {distinct }}(\mathrm{PBC})$ & 1 & 1 & 2 & 4 & 3 & 9 & 14 & $\geq 39$ \\
\hline
\end{tabular}

Table 3: Details about the total number of minima $N_{\min }$ and distinct (non-degenerate) minima $N_{\text {distinct }}$ for the naive functional, trivial orbit, on $N \times N$ lattices.

conjugate gradient search, we find a distribution which is peaked at zero and appears to become more strongly peaked as $N$ is increased. From a practical point of view this suggests that numerical minimisation methods may have a reasonable chance of approaching the fundamental modular region even for larger lattices, without having to explore more than a small fraction of the Gribov copies within the first Gribov region.

\subsection{Stereographical lattice Landau gauge}

We have also attempted to use the MCCGM to find the minima of the 2-D stereographic gauge fixing functional. In this case, we have been restricted to a $3 \times 3$ lattice with APBC, and even there we have not succeeded in finding all the minima, except for the trivial orbit. However, we clearly see that the number of Gribov copies (minima) for the stereographic gauge is larger than the total number of Gribov copies (all stationary points) for the naive lattice Landau gauge. Moreover, the results we have obtained suggest that $n[U]$ for the stereographic gauge is orbit dependent. As an illustration of this, Fig. 10 shows the number of distinct minima found for three random orbits, as a function of the number of initial guesses. We find that the number of Gribov copies is greater than 4500 for both random orbits considered, and 7466 for the trivial orbit. We may compare this with the total number of Gribov copies for the naive gauge shown in Table1, which ranges between 2300 and 2700 for the 10 orbits considered there. This shows that the exponential suppression of the number of Gribov copies observed in 1 dimension does not hold in higher dimensions, and the stereographic gauge is thus less advantageous from this point of view than the naive gauge. Furthermore, the number of minima found for the two random orbits and the trivial do not appear to converge on a single number, although this cannot yet be completely ruled out, as the convergence for the random orbits seems only logarithmic in $N_{\text {guess }}$. Similar results were obtained using the NPHC method for $2 \times 2$ lattices with periodic boundary conditions. 


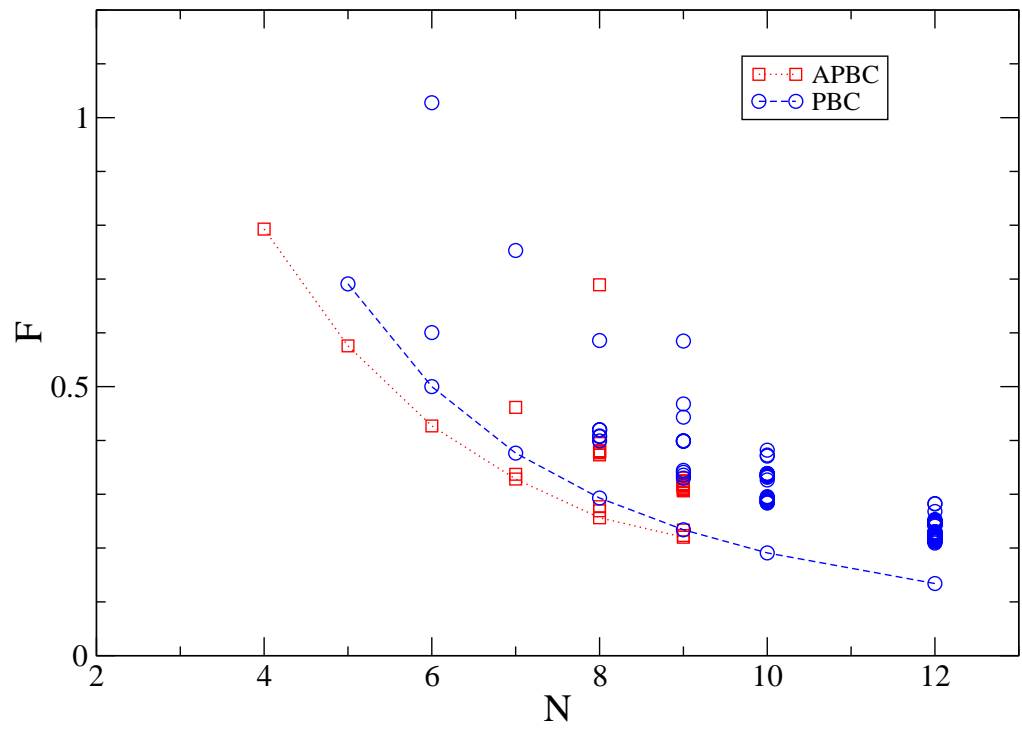

Figure 6: Function values at the minima for the 2-dimensional naive functional, trivial orbit, as a function of the lattice size $N$. Note that the global minima at $F=0$ are not shown here.

\section{Discussion and conclusions}

In this paper, we have studied three alternative definitions of the lattice Landau gauge: minimal lattice Landau gauge, absolute lattice Landau gauge, and the stereographically modified lattice Landau gauge. We have focused on the compact $\mathrm{U}(1)$ case only for this paper, with $\beta=0$.

Our strategy is to first collect (if available) and work out the analytical results for Gribov copies for the one dimensional lattice and, as far as possible, for the higher dimensional lattices. For the one dimensional case, we do have a complete understanding of Gribov copies for the above mentioned gauges. For the two dimensional cases, we have been able to derive some lower bounds on the number of Gribov copies. Then, we use the recently developed numerical polynomial homotopy continuation (NPHC) method to find all Gribov copies for a two dimensional lattice. Here, though we could only solve small lattices of the size $3 \times 3$ completely, we emphasise that this is the first ever result where all Gribov copies are found in more than one dimension. We use the NPHC method to study the orbit-dependence of the number of Gribov copies thoroughly for these lattices. For the bigger lattices, we move to a traditional method, namely, Monte Carlo Conjugate Gradient Method (MCCGM) to attempt to find 'all' minima.

Our findings are summarised below.

1. Minimal lattice Landau gauge: The number of minima for the naive functional in one dimension with antiperiodic boundary conditions is orbit-independent, but in two dimensions it is orbit dependent. For periodic boundary conditions, the number of minima is orbit-dependent in any dimension. 


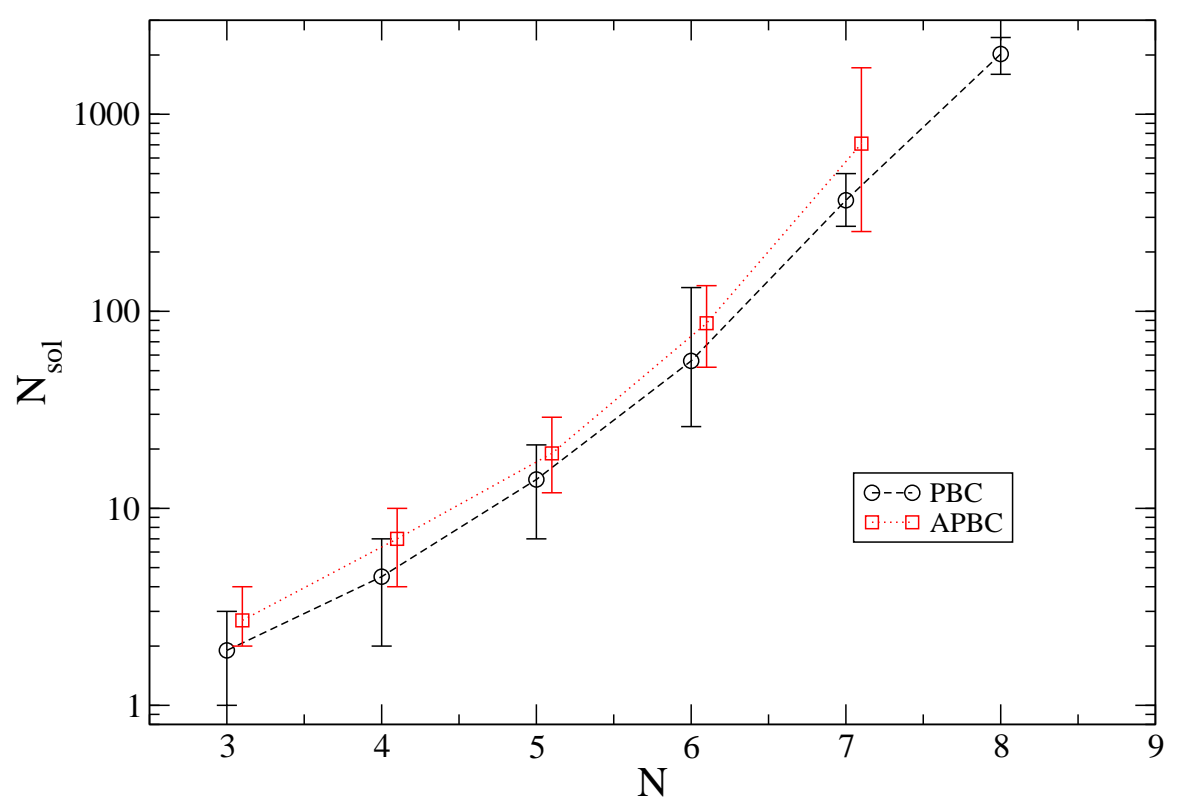

Figure 7: Number of distinct solutions $N_{\text {sol }}$ found for random orbits on $N \times N$ lattices with the naive functional. The data points denote the average number of solutions found for each $N$, while the error bars denote the $68 \%$ confidence intervals (the 16th and 84th percentiles for the number of solutions found). The data for antiperiodic boundary conditions (APBC) are offset horizontally for clarity. The $N=8$ data are taken from only two random orbits.

2. Absolute lattice Landau gauge: The number of global minima for any boundary conditions for the naive functional is orbit-independent, in both one and two dimensions. This holds for both the naive and stereographic gauge fixing functional, in all cases where we have obtained results.

3. Stereographic gauge: We first show that the FP operator for the stereographic gauge is generically positive definite, and hence all the stationary points of the corresponding gauge fixing functional are minima. We find that the number of minima is orbit-independent in one dimension for any boundary conditions, but appears to be orbit-dependent in two dimensions. The number of minima for the stereographic gauge in higher dimensions is much higher than even the number of all the stationary points for the naive functional on the same lattice. This is exactly opposite to the earlier claim based on the one-dimensional results that the number of minima (Gribov copies) is exponentially suppressed for the stereographic gauge case compared to the naive gauge. It is worth noting that the stereographic and naive Landau gauge should become equivalent in the continuum limit and that the difference between the two should be due to lattice artefacts. This may still pose problems for simulations at realistic couplings, however.

4. We have not found any clear evidence for the conjecture that the first Gribov region 

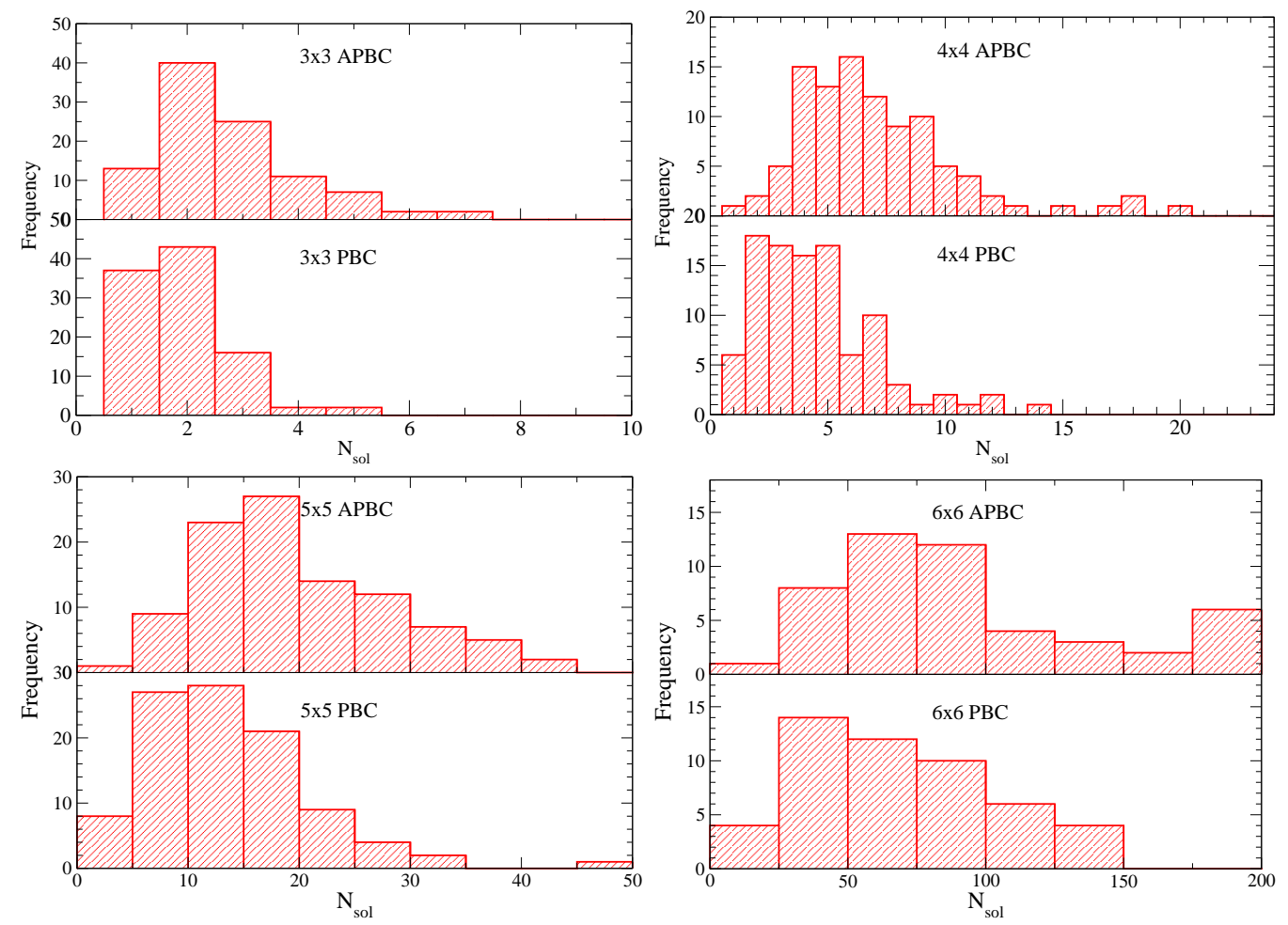

Figure 8: Distribution of the number of distinct minima $N_{\text {sol }}$ found for random orbits with the naive functional on different lattice sizes.

becomes equivalent to the fundamental modular region in the $N \rightarrow \infty$ limit, by studying the distribution of functional values at the minima and their median distance from the global minimum. It may well be that the convergence to the infinite volume limit is so slow that it would not be observed on these small lattices. It is also possible we would need to go beyond strong coupling and approach the continuum limit to see this. That is beyond the scope of this study.

Based on our results, we conclude that it would be too cumbersome to fix a gauge using the Faddeev-Popov procedure on the lattice with averaging over all Gribov copies, due to the orbitdependence of the Gribov copies in the minimal lattice Landau gauge and stereographic gauge. The naive lattice Landau gauge, as is well-known, suffers from the Neuberger $0 / 0$ problem. Only the absolute lattice Landau gauge seems to rescue the situation in that it evades the Neuberger $0 / 0$ problem by construction and the number of Gribov copies (global minima) is orbit independent. However, finding the global minimum of such functions is in general an NP hard problem, and it is only if typical local minima become indistinguishable from the global minimum that this becomes a viable alternative. Although we find no evidence that the function values of the minima as a whole accumulate near the global minimum, we do find that values near the global minimum are more likely to be found. It remains an open question, though, 

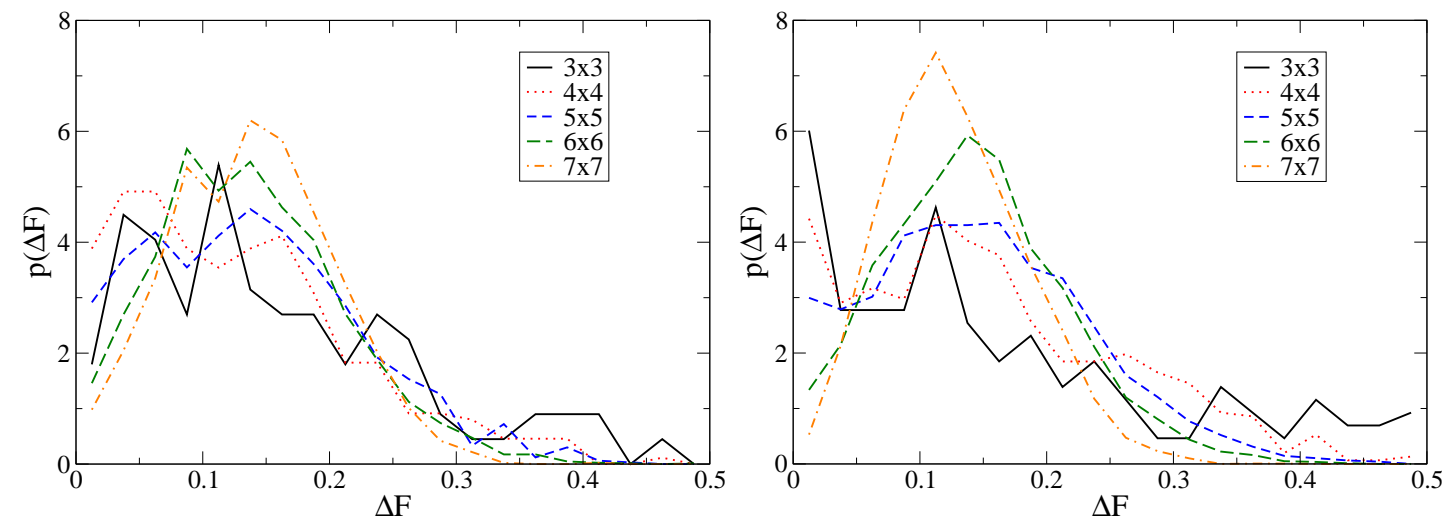

Figure 9: Distribution of the values of the naive gauge fixing functional, $\Delta F=F_{\operatorname{med}}-F_{\min }$, for random orbits on different lattice sizes. Left: periodic boundary conditions; right: antiperiodic boundary conditions.

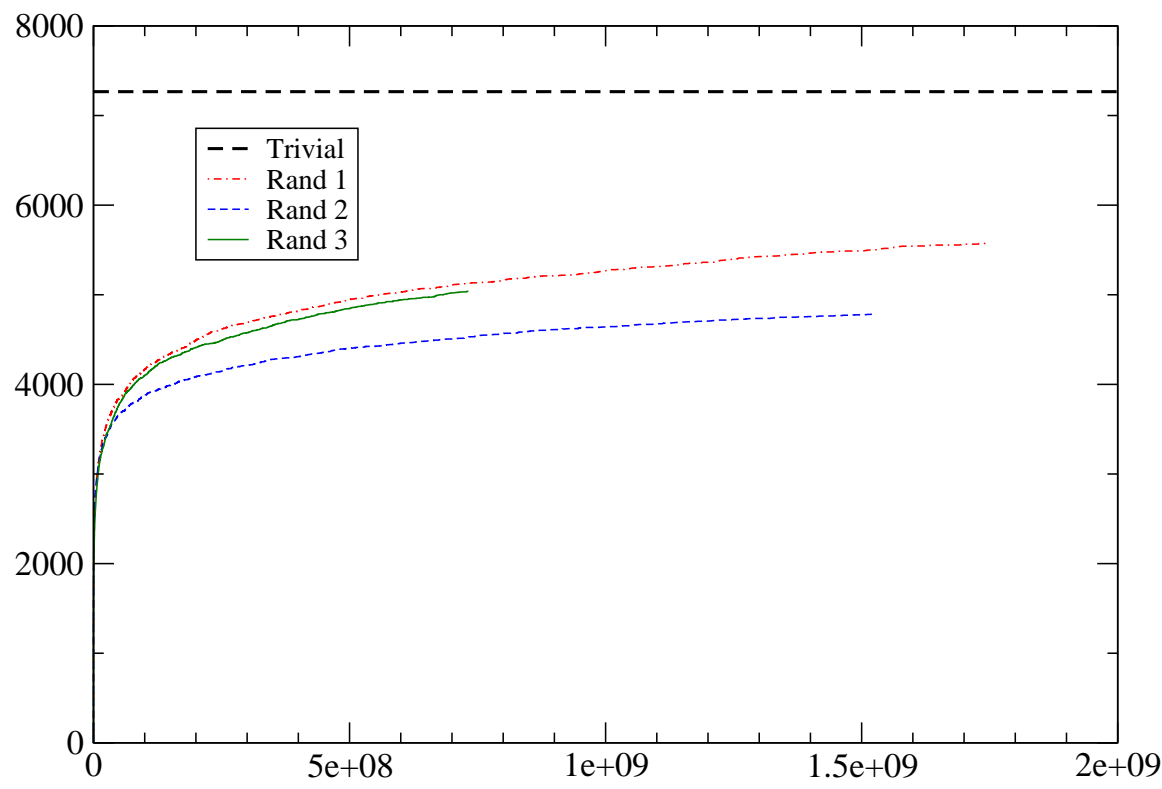

Figure 10: Number of distinct minima found for the stereographic gauge, for 3 different random orbits, as a function of the number of initial guesses $N_{\text {guess }}$. Also shown is the result for the trivial orbit. 
whether this is still the case for larger lattices and other gauge groups. It is also not known whether these near-global minima also exhibit the same qualitative behaviour for quantities such as gauge and ghost propagators as the absolute minimum.

In the context of gauge fixing using a weighted average over Gribov copies as proposed in Refs 40, our results shown in Fig. 9] suggest that the functional values tend to a smooth distribution as $N$ grows. This means that a weighted average would be feasible, unlike for example the case where there was a gap between the global minimum and all other minima, resulting in a potentially serious overlap or ergodicity problem in Monte Carlo simulations. On the other hand, if the results in Sec. 4 hold also for larger lattices (and other gauge groups), then higher order stationary points are exponentially suppressed compared to the minima, so an algorithm that samples only minima (or only some saddle points) may provide a reasonable approximation to the full functional integral.

We should reiterate that Gribov copies in compact $\mathrm{U}(1)$ gauge theory are purely a lattice artefact with no continuum counterpart, while $\mathrm{SU}\left(N_{c}\right)$ gauge theories exhibit continuum Gribov copies on top of those which are lattice artefacts. However, understanding the nature of Gribov copies in compact $\mathrm{U}(1)$ is crucial to a full understanding of Gribov copies in $\mathrm{SU}\left(N_{c}\right)$ theories containing compact $\mathrm{U}(1)$ as a subgroup, and in particular the Neuberger $0 / 0$ problem will be resolved once the problem is resolved for compact $\mathrm{U}(1)$. Our finding that the stereographic gauge does not provide a satisfactory resolution of this problem can therefore be expected to be directly applicable to other gauge groups. The bounds on the number of Gribov copies derived in Appendix $\mathrm{B}$ are also valid for all $\mathrm{SU}\left(N_{c}\right)$ groups.

\section{Acknowledgments}

This work was supported by the U.S. Department of Energy under contract no. DE-FG0285ER40237 and by Science Foundation Ireland grant 08-RFP-PHY1462. CH acknowledges the receipt of a SPUR scholarship. JIS acknowledges the hospitality of the INT at the University of Washington, where this work was completed. A part of this paper is based on DM's thesis and he would like to thank Lorenz von Smekal for numerous discussions about issues related to Gribov copies and the Neuberger 0/0 problem. DM would also like to thank Anthony Williams for his kind support and discussions. We would like to thank André Sternbeck for his valuable feedback and insights specially on the numerical issues related to Gribov copies.

\section{A Absence of Neuberger problem in stereographic gauge}

Here we sketch the proof of the statement that there is no Neuberger $0 / 0$ problem for the steregraphic gauge in any dimension with either APBC or PBC. Essentially, the proof boils down to showing that the corresponding FP operator is generically a positive definite matrix and hence there is no cancellation among signs of the FP determinants at Gribov copies.

We first note that the matrix $M_{F P}^{s}$ corresponds to a hessian matrix of a functional with nearestneighbour coupling so it can be decomposed using projection matrices (i.e., matrices with entries in $\{-1,0,1\}$ ) with only two entries per row (or column). Thus, with APBC, the FP operator 
for the stereographic gauge Eq. (14) can be decomposed as

$$
M_{F P}^{s}=\sum_{\mu=1}^{d}\left(M_{\mu} D_{\mu}^{s} M_{\mu}^{T}\right),
$$

where $D_{\mu}^{s}$ are diagonal matrices with the diagonal entries $\sec ^{2}\left(\left(\phi_{i, \mu}+\theta_{i+\hat{\mu}}-\theta_{i}\right) / 2\right)$ with $i$ running over all lattice sites and $\mu$ running over the lattice dimension $d$. Here, the matrices $M_{\mu}$ consist of $\{-1,0,1\}$ as their entries, i.e., the $i$-th row consists of entries corresponding to the nearest-neighbour interaction at the $i$-th site in the $\mu$-th direction. For the APBC case, $M_{\mu}$ are non-singular matrices because we have removed the global gauge degree of freedom using the boundary condition. Since $M_{\mu} D_{\mu}^{s} M_{\mu}^{T}$ are positive definite matrices for all $\mu=1, \ldots, d$, according to Sylvester's law of inertia (which states that if $A=C B C^{T}$ where $A$ is a real symmetric matrix and $C$ and $B$ are real matrices with $B$ diagonal, then the number of positive, negative and zero eigenvalues of $A$ is the same as those of $B$ ), and because the sum of positive definite matrices is also a positive definite matrix, $M_{F P}^{s}$ is strictly a generically positive definite matrix. Therefore, there is no cancellation of signs of the corresponding FP determinants, and hence, no Neuberger $0 / 0$ problem.

With PBC, a decomposition of the corresponding $M_{F P}^{s}$ is more involved so we use another method here. We first separate $M_{F P}^{s}$ for different $\mu$ in Eq. (14), i.e., $M_{F P}^{s}=\sum_{\mu}\left(M_{F P}^{s}\right)_{\mu}$ for a $d$-dimensional square lattice. Now, we consider the quadratic form for the symmetric matrices $\left(M_{F P}^{s}\right)_{\mu}$ with an $N^{d} \times 1$ vector $\vec{y} \neq \overrightarrow{0}$ whose elements are $y_{i}$ where $i=\left(i_{1}, \ldots, i_{d}\right)$ with $i_{1}, \ldots, i_{d}$ running over $1, \ldots, N$ for the square lattice. Thus, it is straightforward to check that for any $\mu$,

$$
\vec{y}^{T}\left(M_{F P}^{s}\right)_{\mu} \vec{y}=\sum_{i} \sec ^{2} \frac{\phi_{i, \mu}^{\theta}}{2}\left(y_{i+\hat{\mu}}-y_{i}\right)^{2},
$$

which is 0 only if all $y_{i}$ are equal, which is the constant zero mode and is strictly positive for all other cases. Also, these $y_{i}$ are taken to be following the PBC, in this expression. Thus, the matrix $\left(M_{F P}^{s}\right)_{\mu}$ is positive semi-definite for all $\mu=1, \ldots, d$. Since the sum of positive semidefinite matrices is a positive semi-definite matrix, $M_{F P}^{s}$ is also a positive semi-definite matrix. Once we remove the global gauge degree of freedom by taking one of the angles to be zero, say $\theta_{(N, \ldots, N)}=0$, then $M_{F P}$ is strictly positive definite matrix. Thus there is no Neuberger $0 / 0$ in the PBC case either.

\section{B Lower bounds on the number of Gribov copies}

Here, we show how the Morse theoretical interpretation of the lattice Landau gauge can give a lower bound on the number of Gribov copies $n$. We briefly explain the Morse indices, Betti numbers and their relationship with $\chi(\mathbb{M})$ via the Poincaré polynomial $P(z)$ of $\mathbb{M}$ along with Morse inequalities. We then obtain an expression for the lower bound on $n$ at a given orbit and calculate 4 it explicitly for compact $\mathrm{U}(1), \mathrm{SU}(2)$ and $\mathrm{SU}\left(N_{c}\right)$. The ultimate goal of this discussion is to get a generic (orbit-independent) lower bound on $n$ for the naive and stereographic gauge to get a guide on our numerical results, so we ignore the explicit orbit-dependence here.

\footnotetext{
${ }^{4}$ Here, we omit the explicit mention of the orbit-dependence of $n$.
} 
Let $K_{i}$ be the number of critical points of a height function $h(\vec{x})$ with its Hessian at these critical points having $i$ negative eigenvalues. Then $\chi(\mathbb{M})=\sum_{i}(-1)^{i} K_{i}$, where now the sum over $i$ runs from 0 to the dimension of $\mathbb{M}$.

In addition to the Euler characteristic, the Betti numbers are closely related topological invariants of a manifold. Firstly, a homology group is a measure of the hole structure of a manifold, or more specifically, a topological space. There may be several homology groups of a manifold. The $i$ th Betti number $b_{i}$ is defined as the rank of the $i$ th homology group (the reader is referred to [66] for details on Betti numbers and their relation to the Euler characteristic).

The Poincaré polynomial is defined as $P(z)=\sum_{i} b_{i} z^{i}$ for an arbitrary real variable $z$. Then, it turns out that

$$
P(1)=\sum_{i} b_{i}, P(-1)=\sum_{i}(-1)^{i} b_{i}=\chi(\mathbb{M}),
$$

where the sum runs from 0 to the dimension of $\mathbb{M}$.

There are two types of inequalities, called Morse inequalities, which relate $b_{i}$ and $K_{i}$. The weak Morse inequality states that

$$
\sum_{i} K_{i} \geq \sum_{i} b_{i}
$$

and the strong inequality states that

$$
K_{i} \geq b_{i}
$$

for all $i$. Due to the weak Morse inequality and using the fact that $\sum_{i} K_{i}$ is the total number of critical points which is $n$ for a lattice Landau gauge fixing functional, we have

$$
P(1)=\sum_{i} b_{i} \leq \sum_{i} K_{i}=n
$$

where for a $d$-dimensional lattice the sum runs over all the lattice sites, i.e., $i=0, \ldots, N^{d}$ for the APBC case and $i=0, \ldots, N^{d}-1$ for the PBC case. Thus $P(1)$ is a lower bound on $n$. It now remains to calculate $P(z)$ and hence ultimately $P(1)$ for the gauge-group manifold.

To calculate the corresponding $P(z)$, first note that $P_{X \times Y}(z)=P_{X}(z) P_{Y}(z)$ for a product space manifold $X \times Y$ of $X$ and $Y$, where $P_{X}(z)$ and $P_{Y}(z)$ are the Poincaré polynomials of $X$ and $Y$ respectively. For the naive lattice Landau gauge for the compact U(1) case with APBC, the corresponding manifold is $\left(S^{1}\right)^{N^{d}}$ and the corresponding Poincaré polynomial is

$$
\begin{aligned}
P_{\left(S^{1}\right)^{N^{d}}}(z) & =\prod_{k=1}^{N^{d}} P_{S^{1}}(z)=\prod_{k=1}^{N^{d}}(1+z)=(1+z)^{N^{d}}=\sum_{i=0}^{N^{d}}\left(\begin{array}{c}
N^{d} \\
i
\end{array}\right) z^{i} \\
\therefore P_{\left(S^{1}\right)^{N^{d}}}(1) & =2^{N^{d}} \leq \sum_{i=0}^{N^{d}} K_{i}=n,
\end{aligned}
$$

where we have used the fact that $P_{S^{1}}(z)=1+z$, with the Betti numbers $b_{0}=1=b_{1}$ and all others being zero for $S^{1}$. Thus, $n$ must be greater than or equal to $2^{N^{d}}$ in this case. Moreover, we can verify that $P(-1)=(1-1)^{N^{d}}=0=\chi\left(\left(S^{1}\right)^{N^{d}}\right)$ as expected. Also, we identify the Betti 
numbers of this $N^{d}$-torus as $b_{i}=\left(\begin{array}{c}N^{d} \\ i\end{array}\right)$, for all $i=0, \ldots, N$, by comparing the above equation with Eq. (35). Similarly, for the PBC case, the corresponding manifold is $\left(S^{1}\right)^{N^{d}-1}$, i.e.,

$$
\begin{aligned}
P_{\left(S^{1}\right)^{\left(N^{d}-1\right)}}(z) & =\prod_{k=1}^{N^{d}-1} P_{S^{1}}(z)=\prod_{k=1}^{N^{d}-1}(1+z)=\sum_{i=0}^{N^{d}-1}\left(\begin{array}{c}
N^{d}-1 \\
i
\end{array}\right) z^{i} \\
\therefore P_{\left(S^{1}\right)^{\left(N^{d}-1\right)}}(1) & =2^{N^{d}-1} \leq \sum_{i=0}^{N^{d}-1} K_{i}=n .
\end{aligned}
$$

So, the corresponding Betti numbers for $\left(S^{1}\right)^{N^{d}-1}$ are $b_{i}=\left(\begin{array}{c}N^{d}-1 \\ i\end{array}\right)$ for $i=0, \ldots, N^{d}-1$.

For $\mathrm{SU}(2)$, the group manifold is $S^{3}$ and the corresponding Poincaré polynomial is $P_{S^{3}}(z)=$ $\left(1+z^{3}\right)$. Thus, for the naive lattice Landau gauge with PBC the corresponding Poincaré polynomial is

$$
\begin{aligned}
P_{\left(S^{3}\right)^{(N-1)}}(z) & =\prod_{k=1}^{N-1}\left(1+z^{3}\right)=\left(1+z^{3}\right)^{N^{d}-1} \\
\therefore P_{\left(S^{3}\right)^{\left(N^{d}-1\right)}}(1) & =2^{N^{d}-1} \leq n^{S U(2)},
\end{aligned}
$$

giving the same lower bound for the number of Gribov copies as that of the compact U(1) case. For a generic $\mathrm{SU}\left(N_{c}\right)$ with $N_{c}>2$, the group manifold is $S^{3} \times S^{5} \times \ldots S^{2 N_{c}-1}$ and the corresponding Poincaré polynomial is $\left(1+z^{3}\right)\left(1+z^{5}\right) \ldots\left(1+z^{2 N-1}\right)$. Thus, for the naive lattice Landau gauge with $\mathrm{PBC}$,

$$
\begin{aligned}
P_{\left(S^{3} \times \cdots \times S^{2 N_{c}-1}\right)^{\left(N^{d}-1\right)}}(z) & =\left(\left(1+z^{3}\right)\left(1+z^{5}\right) \ldots\left(1+z^{2 N_{c}-1}\right)\right)^{N^{d}-1} \\
\therefore P_{\left(S^{3} \times \cdots \times S^{2 N_{c}-1}\right)^{\left(N^{d}-1\right)}}(1) & =2^{\left(N_{c}-1\right)\left(N^{d}-1\right)} \leq n^{S U\left(N_{c}\right)},
\end{aligned}
$$

giving a larger lower bound than that of the compact $\mathrm{U}(1)$ and $\mathrm{SU}(2)$ cases.

On the other hand, the corresponding manifold for the stereographic gauge is $\mathbb{R}^{N^{d}}$ for the compact $\mathrm{U}(1)$ case with APBC. Here, $b_{0}=1$, for $\mathbb{R}$, and all other $b_{i}=0$ for $i=1, \ldots, N$. So the corresponding Poincaré polynomial, with $P_{\mathbb{R}}(z)=1$, is

$$
\begin{aligned}
P_{\mathbb{R}^{N^{d}}}(z) & =\prod_{k=1}^{N^{d}}(1)=1^{N^{d}}=1 \\
\therefore P_{\mathbb{R}^{N^{d}}}(-1) & =P_{\mathbb{R}^{N^{d}}}(1)=1 \leq n .
\end{aligned}
$$

Thus, the lower bound on the number of Gribov copies is exponentially suppressed from $2^{N^{d}}$ for the naive gauge to 1 in the stereographic gauge for compact U(1). Similarly, with PBC,

$$
\begin{aligned}
P_{\mathbb{R}^{N^{d}-1}}(z) & =\prod_{k=1}^{N^{d}-1}(1)=1^{N^{d}-1}=1 \\
\therefore P_{\mathbb{R}^{N^{d}-1}}(-1) & =P_{\left(\mathbb{R}^{N^{d}-1}\right.}(1)=1 \leq n .
\end{aligned}
$$

Furthermore, the corresponding Betti numbers of $\mathbb{R}^{N-1}$ are $b_{0}=1$ and $b_{i}=0$ for $i=1, \ldots, N$ for the APBC case and $b_{0}=1$ and $b_{i}=0$ with $i=1, \ldots, N^{d}-1$ for the PBC case. 


\section{Gauge-fixing functional at minima}

Here we want to describe the function values at minima of the gauge-fixing functional in the infinite volume limit.

In one dimension, we have the complete classification of Gribov copies for the naive Landau gauge with $\mathrm{PBC}$ and odd $N$, given in Eq. (20): the minima satisfy

$$
\phi_{i}^{\theta}=\phi_{N}^{\theta}=\bar{\phi}+\frac{2 \pi l}{N} \quad \bmod 2 \pi, \quad l \in\{0, \ldots, N-1\}, \quad \text { with } \quad \bar{\phi} \equiv \frac{\sum_{i=1}^{N} \phi_{i}}{N}
$$

together with the condition $\cos \phi_{N}^{\theta}>0$. The functional value at the minima is then $\left.F_{\phi}(\theta)\right|_{\text {minima }}=$ $\left(1-\cos \phi_{N}^{\theta}\right)$.

By the law of large numbers, since each $\phi_{i}$ is independent and identically distributed, the mean value $\bar{\phi} \rightarrow 0$ as $N \rightarrow \infty$. Assuming that $N$ is large enough to ignore the $\bar{\phi}$ term, we notice that the stationary points with a random orbit in the thermodynamic limit are the same as those for the trivial orbit. Hence in the thermodynamic limit, the first Gribov region of every random orbit is identical to the first Gribov region of the trivial orbit. This is a surprising result since for finite $N$, the Gribov regions are orbit dependent.

In the limit where $\bar{\phi} \rightarrow 0$, the condition $\cos \phi_{N}^{\theta}>0$ becomes equivalent to $l \leq \frac{N}{4}$ or $l \geq \frac{3 N}{4}$. Since the function values are the same for minima in $l \in\left\{0, \ldots, \frac{N}{4}\right\}$ and $l \in\left\{\frac{3 N}{4}, \ldots, N-1\right\}$, let us choose $l \in\left\{0, \ldots, \frac{N}{4}\right\}$. Computing $F=1-\cos (2 \pi l / N)$ gives us values in the interval $[0,1]$. We have partitioned $[0,1]$ into $\frac{N}{4}+1$ pieces (the size of the set $\left\{0, \ldots, \frac{N}{4}\right\}$ ). Call the value of the $i$ 'th piece $p_{i}$, where $p_{i}$ corresponds to the function value of $l=i$. For sufficiently large $N$, the set of all $p_{i}$ will become a countably infinite set obtaining values that appear to map the graph of $F(x)=1-\cos (x)$ for $0 \leq x \leq \frac{\pi}{2}$.

However, for values of $l \ll N, \cos (2 \pi l / N) \approx 1$ and $F \approx 0$. In our partition of $[0,1]$ this is equivalent to $\left[0, p_{k}\right] \rightarrow 0$ as $N \rightarrow \infty$ for a particular $p_{k}$. As $N$ is assumed large, the rest of the interval stretches towards zero because we divide by $N$. Hence the function value of zero is hugely degenerate for $N$ sufficiently large with all other function values that are not sufficiently near the origin being unique. We now take into account the other interval $l \in\left\{\frac{3 N}{4}, \ldots, N-1\right\}$ which has the same function values as the first interval. Therefore $F=0$ is hugely degenerate and all other function values not sufficiently close to the origin are 2-fold degenerate. Hence in the thermodynamic limit, minima occur taking function values between $[0,1]$ but zero occurs dramatically more often.

This argument is supported by the numerical results shown in Figure 4. We see that even for small lattices sizes, the function values at the minima start to accumulate near zero.

For the naive Landau gauge with PBC in two dimensions, let $\theta$ be a stationary point. Assume that this satisfies some conditions $c_{i, j}=\phi_{i+1, j, x}+\theta_{i+1, j}-\theta_{i, j}$ and $d_{i, j}=\phi_{i, j+1, y}+\theta_{i, j+1}-\theta_{i, j}$. Then the gradient equations become

$$
\sin \left(c_{i+1, j}\right)-\sin \left(c_{i, j}\right)+\sin \left(d_{i, j+1}\right)-\sin \left(d_{i, j}\right)=0 .
$$

Let us now assume that $c_{i, j}, d_{i, j}$ satisfy the one dimensional gradient equations

$$
\begin{aligned}
\sin \left(d_{i, j+1}\right)-\sin \left(d_{i, j}\right) & =0, \\
\sin \left(c_{i+1, j}\right)-\sin \left(c_{i, j}\right) & =0 .
\end{aligned}
$$


Then these satisfy the 2-dimensional gradient equations, and we have found stationary points for which the gradient equations decouple into lower dimensional ones. For a particular orbit, we may then find a higher dimensional stationary point by solving the one dimensional gradient equations for each hypersurface with $j$ fixed in $c_{i, j}$ and $i$ fixed in $d_{i, j}$. However, optimising the one dimensional gradient equations for $c_{i, j}$ with $j$ fixed fixes the $j$ 'th column of lattice sites. With $j \in\{1, \ldots, N\}$, this fixes all lattice sites. Therefore, we cannot vary the $\theta_{i, j}$ in order to optimise $d_{i, j}$ for fixed $i$ with a random orbit. We must impose the trivial orbit. The gradient equations are solved only if $d_{i, j+1}=c_{G(i+1, j)}$ where $G$ is some lattice symmetry which imposes $\sin \left(d_{i, j+1}\right)=\sin \left(d_{i, j}\right)$ so that the gradient equations are simultaneously optimised. Now we can optimise each hypersurface $c_{i, j}$ for fixed $j$ but it is only the subset of these SPs which simultaneously imposes the gradient equations of $d_{i, j}$ that satisfy the two dimensional gradient equations. Using the analytic formula for one dimensional SPs with the trivial orbit from section 3.1. we can find these two dimensional SPs. As noted, the minima occur at all $q_{k}=0$ and thus the two dimensional minima we can construct are

$$
c_{i, j}=\frac{2 \pi l}{N}, \quad d_{i, j}=c_{G(i, j)}=\frac{2 \pi G(l)}{N},
$$

where $l \in\left\{0, \ldots, \frac{N}{4}, \frac{3 N}{4}, \ldots, N-1\right\}$.

We can explicitly construct some of these minima. With the above conventions, first note that requiring $d_{i, j}=0(G(l)=0)$ simultaneously solves the gradient equations for each $l$. This is easily visualised by first fixing a lattice site to impose boundary conditions and then layering a fixed hypersurface of a 1-dimensional SP in a constant direction until a 2-dimensional lattice is filled. Putting this back into the definition of $F$ it is easy to see that, in this specific case, the two dimensional minima has the same function value as the one dimensional minima. The Hessian (FP operator) in this $G(l)=0$ case is given by

$$
M_{F P}=\frac{\partial^{2} F}{\partial \theta_{i, j} \partial \theta_{k, l}}=\left(M_{F P}^{1 D}\right)_{i, k} \delta_{j, l}-\left(\delta_{j+1, l}+\delta_{j-1, l}-2 \delta_{j, l}\right) \delta_{i, k}
$$

where $M_{F P}^{1 D}$ is the Hessian of the one dimensional minima, which is a $N \times N$ matrix. From (48) we see that $M_{F P}^{1 D}+2 I$ occur in blocks along the main diagonal. The other non zero terms come from the second term in (48). They are upper and lower diagonal -1 terms starting at the $(1, N+1)$ and $(N+1,1)$ matrix element. Boundary conditions give diagonal -1 terms starting at the $\left(1, N^{2}-N\right)$ and $\left(N^{2}-N, 1\right)$ matrix element. To fully impose PBC we must remove one linearly dependent gradient equation. This reduces the Hessian to a $\left(N^{2}-1\right) \times\left(N^{2}-1\right)$ matrix. These minima were observed numerically. More minima can be constructed by defining $G$ and we can find others by the permutation symmetry as described in section 3.2 . Therefore we can construct multiple sets of two dimensional minima were each set is in a one-to-one correspondence with the set of all one dimensional minima.

Since we can construct a number of 2-dimensional minima from each one dimensional minimum, the two dimensional first Gribov region will contain multiple analogues of the one dimensional first Gribov region. By the above arguments, since most function values of the 1-dimensional minima accumulate at zero in the thermodynamic limit, this behaviour must be imitated locally by the 2 -dimensional first Gribov region. As this construction of minima can be extended to higher dimensions, this argument generalises. 
We cannot comment on the global behaviour of the two dimensional first Gribov region as an analytic classification of all two dimensional minima remains elusive. However, the numerical results from Figure 6 show that the function values of 2-dimensional minima with a trivial orbit appear to decay towards zero, similar to the one dimensional case. This suggests that the imitation may be global for the trivial orbit. Since this construction only works for the trivial orbit, there is no reason to suggest that the two dimensional first Gribov region for a random orbit behaves locally like the one dimensional first Gribov region. Indeed, this difference between the trivial and random orbit case was observed in section 5.2.1 and particularly in Table 4.

\section{References}

[1] H. J. Rothe, Lattice gauge theories: An introduction, World Sci. Lect. Notes Phys. 74 (2005) 1-605.

[2] J. Smit, Introduction to quantum fields on a lattice: A robust mate, Cambridge Lect. Notes Phys. 15 (2002) 1-271.

[3] I. Montvay and G. Münster, Quantum fields on a lattice, . Cambridge, UK: Univ. Pr. (1994) 491 p. (Cambridge monographs on mathematical physics).

[4] R. Alkofer and L. von Smekal, The infrared behavior of QCD green's functions: Confinement, dynamical symmetry breaking, and hadrons as relativistic bound states, Phys. Rept. 353 (2001) 281 arXiv:hep-ph/0007355.

[5] L. D. Faddeev and V. N. Popov, Feynman diagrams for the Yang-Mills field, Phys. Lett. B25 (1967) 29-30.

[6] C. Becchi, A. Rouet and R. Stora, Renormalization of gauge theories, Annals Phys. 98 (1976) 287-321.

[7] V. N. Gribov, Quantization of non-Abelian gauge theories, Nucl. Phys. B139 (1978) 1.

[8] I. M. Singer, Some remarks on the Gribov ambiguity, Commun. Math. Phys. 60 (1978) $7-12$.

[9] H. Neuberger, Nonperturbative BRS invariance, Phys. Lett. B175 (1986) 69.

[10] H. Neuberger, Nonperturbative BRS invariance and the Gribov problem, Phys. Lett. B183 (1987) 337.

[11] K. Fujikawa, Path integral measure for gauge invariant fermion theories, Phys. Rev. Lett. 42 (1979) 1195.

[12] P. Hirschfeld, Strong evidence that Gribov copying does not affect gauge theory functional integral, Nucl. Phys. B157 (1979) 37.

[13] B. Sharpe, Gribov copies and the Faddeev-Popov formula in lattice gauge theories, J.Math.Phys. 25 (1984) 3324-3330. 
[14] L. von Smekal, D. Mehta, A. Sternbeck and A. G. Williams, Modified lattice Landau gauge, PoS LAT2007 (2007) 382 arXiv:0710.2410.

[15] L. von Smekal, A. Jorkowski, D. Mehta and A. Sternbeck, Lattice Landau gauge via stereographic projection, PoS CONFINEMENT8 (2008) 048 [arXiv:0812.2992].

[16] L. von Smekal, Landau gauge QCD: Functional methods versus lattice simulations, arXiv:0812.0654

[17] R. Aouane, V. Bornyakov, E.-M. Ilgenfritz, V. Mitrjushkin, M. Müller-Preussker et. al., Landau gauge gluon and ghost propagators at finite temperature from quenched lattice QCD, Phys.Rev. D85 (2012) 034501 [arXiv:1108.1735].

[18] V. Bornyakov, V. Mitrjushkin and R. Rogalyov, Gluon propagators in 3d SU(2) theory and effects of Gribov copies, Phys.Rev. D86 (2012) 114503 [arXiv:1112.4975].

[19] A. Cucchieri, Gribov copies in the minimal Landau gauge: The influence on gluon and ghost propagators, Nucl. Phys. B508 (1997) 353-370 arXiv:hep-lat/9705005.

[20] B. Holdom, Gribov copies and anomalous scaling, Phys. Rev. D78 (2008) 125030 arXiv:0712.2379.

[21] B. Holdom, Counting Gribov copies, Phys. Rev. D79 (2009) 085013 arXiv:0901.0497.

[22] A. Ilderton, Gribov copies and confinement, eConf C0706044 (2007) 28 arXiv:0709.1671.

[23] A. Ilderton, M. Lavelle and D. McMullan, Colour, copies and confinement, JHEP 0703 (2007) 044 arXiv:hep-th/0701168.

[24] G. Dell'Antonio and D. Zwanziger, Every gauge orbit passes inside the Gribov horizon, Commun. Math. Phys. 138 (1991) 291-299.

[25] M. Schaden, Equivariant gauge fixing of SU(2) lattice gauge theory, Phys. Rev. D59 (1999) 014508 arXiv:hep-lat/9805020.

[26] D. Zwanziger, Local and renormalizable action from the Gribov horizon, Nucl. Phys. B323 (1989) 513-544.

[27] N. Vandersickel and D. Zwanziger, The Gribov problem and QCD dynamics, Phys.Rept. 520 (2012) 175-251 arXiv:1202.1491].

[28] D. Mehta, Lattice vs. continuum: Landau gauge fixing and 't Hooft-Polyakov monopoles, Ph.D. Thesis, Adelaide University (2009).

[29] D. Mehta and M. Kastner, Stationary point analysis of the one-dimensional lattice Landau gauge fixing functional, aka random phase xy hamiltonian, Annals Phys. $\mathbf{3 2 6}$ (2011) 1425-1440 arXiv:1010.5335.

[30] D. Zwanziger, Fundamental modular region, Boltzmann factor and area law in lattice gauge theory, Nucl. Phys. B412 (1994) 657-730. 
[31] P. van Baal, Gribov ambiguities and the fundamental domain, arXiv:hep-th/9711070.

[32] A. Sternbeck, E.-M. Ilgenfritz, M. Müller-Preussker and A. Schiller, Towards the infrared limit in SU(3) Landau gauge lattice gluodynamics, Phys. Rev. D72 (2005) 014507 arXiv:hep-lat/0506007.

[33] S. Catterall, D. B. Kaplan and M. Ünsal, Exact lattice supersymmetry, Phys. Rept. 484 (2009) 71-130 arXiv:0903.4881.

[34] S. Catterall, R. Galvez, A. Joseph and D. Mehta, On the sign problem in 2d lattice super Yang-Mills, JHEP 1201 (2012) 108 arXiv:1112.3588].

[35] R. Galvez, S. Catterall, A. Joseph and D. Mehta, Investigating the sign problem for two-dimensional $\mathcal{N}=(2,2)$ and $\mathcal{N}=(8,8)$ lattice super Yang-Mills theories, PoS LATTICE2011 (2011) 064 [arXiv:1201.1924.

[36] D. Mehta, S. Catterall, R. Galvez and A. Joseph, Supersymmetric gauge theories on the lattice: Pfaffian phases and the Neuberger 0/0 problem, PoS LATTICE2011 (2011) 078 arXiv:1112.5413.

[37] C. Parrinello and G. Jona-Lasinio, A modified Faddeev-Popov formula and the Gribov ambiguity, Phys.Lett. B251 (1990) 175-180.

[38] D. Zwanziger, Quantization of gauge fields, classical gauge invariance and gluon confinement, Nucl. Phys. B345 (1990) 461-471.

[39] S. Fachin and C. Parrinello, Global gauge fixing in lattice gauge theories, Phys. Rev. D44 (1991) 2558-2564.

[40] J. Serreau and M. Tissier, Lifting the Gribov ambiguity in Yang-Mills theories, Phys.Lett. B712 (2012) 97-103 arXiv:1202.3432.

[41] UKQCD Collaboration, D. Henty, O. Oliveira, C. Parrinello and S. Ryan, Soft covariant gauges on the lattice, Phys. Rev. D54 (1996) 6923-6927 arXiv:hep-lat/9607014.

[42] A. Maas, Constructing non-perturbative gauges using correlation functions, Phys.Lett. B689 (2010) 107-111 arXiv:0907.5185.

[43] A. C. Kalloniatis, L. von Smekal and A. G. Williams, Curci-Ferrari mass and the Neuberger problem, Phys. Lett. B609 (2005) 424-429 arXiv:hep-lat/0501016.

[44] M. Ghiotti, L. von Smekal and A. G. Williams, Extended double lattice BRST, Curci-Ferrari mass and the Neuberger problem, AIP Conf. Proc. 892 (2007) 180-182 arXiv:hep-th/0611058.

[45] L. von Smekal, M. Ghiotti and A. G. Williams, Decontracted double BRST on the lattice, Phys. Rev. D78 (2008) 085016 arXiv:0807.0480.

[46] A. Maas, Describing gauge bosons at zero and finite temperature, arXiv:1106.3942.

[47] J. E. Hetrick, P. de Forcrand, A. Nakamura and R. Sinclair, Son of gauge fixing on the lattice, Nucl. Phys. Proc. Suppl. 26 (1992) 432-434. 
[48] Private communication with Lorenz von Smekal.

[49] A. J. Sommese and C. W. Wampler, The numerical solution of systems of polynomials arising in Engineering and Science. World Scientific Publishing Company, 2005.

[50] D. Mehta, A. Sternbeck, L. von Smekal and A. G. Williams, Lattice Landau gauge and algebraic geometry, PoS QCD-TNT09 (2009) 025 arXiv:0912.0450.

[51] D. Mehta, Finding all the stationary points of a potential energy landscape via numerical polynomial homotopy continuation method, Phys.Rev. E84 (2011) 025702

arXiv:1104.5497.

[52] D. Mehta, Numerical polynomial homotopy continuation method and string vacua, Adv. High Energy Phys. 2011 (2011) 263937 arXiv:1108.1201.

[53] M. Kastner and D. Mehta, Phase transitions detached from stationary points of the energy landscape, Phys. Rev. Lett. 107 (2011) 160602 [arXiv:1108.2345].

[54] R. Nerattini, M. Kastner, D. Mehta and L. Casetti, Exploring the energy landscape of XY models, arXiv:1211.4800.

[55] D. Mehta, J. D. Hauenstein and M. Kastner, Energy landscape analysis of the two-dimensional nearest-neighbor $\phi^{4}$ model, Phys. Rev. E85 (2012) 061103 arXiv:1202.3320.

[56] M. Maniatis and D. Mehta, Minimizing Higgs potentials via numerical polynomial homotopy continuation, Eur. Phys. J. Plus 127 (2012) 91 arXiv:1203.0409.

[57] D. Mehta, Y.-H. He and J. D. Hauenstein, Numerical algebraic geometry: A new perspective on string and gauge theories, JHEP 1207 (2012) 018 [arXiv:1203.4235.

[58] J. Hauenstein, Y.-H. He and D. Mehta, Numerical analyses on moduli space of vacua, arXiv: 1210.6038

[59] T. Y. Li, Solving polynomial systems by the homotopy continuation method, Handbook of numerical analysis XI (2003) 209-304.

[60] D. J. Bates, J. D. Hauenstein, A. J. Sommese and C. W. Wampler. Available at http://www.nd.edu/ sommese/bertini.

[61] J. Verschelde, Algorithm 795: PHCpack: a general-purpose solver for polynomial systems by homotopy continuation, ACM Trans. Math. Softw. 25 (1999), no. 2 251-276.

[62] T. Gunji, S. Kim, M. Kojima, A. Takeda, K. Fujisawa and T. Mizutani, PHoM - a polyhedral homotopy continuation method for polynomial systems, Computing 73 (2004), no. 1 57-77.

[63] T. Gao, T. Y. Li and M. Wu, Algorithm 846: MixedVol: a software package for mixed-volume computation, ACM Trans. Math. Softw. 31 (2005), no. 4 555-560. 
[64] T. Ojika, S. Watanabe and T. Mitsui, Deflation algorithm for the multiple roots of a system of nonlinear equations, Journal of Mathematical Analysis and Applications 96 (1983), no. 2 463-479.

[65] W. T. Vetterling, W. H. Press, S. A. Teukolsky and B. P. Flannery, Numerical Recipes. Cambridge University Press, 3 ed., 2007.

[66] D. Birmingham, M. Blau, M. Rakowski and G. Thompson, Topological field theory, Phys. Rept. 209 (1991) 129-340. 


\begin{tabular}{|l|lllll|}
\hline$N$ & 3 & 4 & 5 & 6 & 7 \\
\hline$N_{\text {orb }}$ & 100 & 100 & 100 & 50 & 10 \\
$N_{\text {guess }}$ & $2 \cdot 10^{4}$ & $5 \cdot 10^{4}$ & $2 \cdot 10^{5}$ & $2 \cdot 10^{6}$ & $6 \cdot 10^{7}$ \\
\hline \multicolumn{5}{|c|}{ Periodic boundary conditions } \\
\hline$\left\langle N_{\text {sol }}\right\rangle$ & 1.9 & 4.5 & 14 & 70 & 373 \\
$68 \%$ CI & $1-3$ & $2-7$ & $7-21$ & $40-111$ & $276-534$ \\
$\left\langle F_{\text {min }}\right\rangle$ & $0.56_{-12}^{+13}$ & $0.48_{-8}^{+8}$ & $0.44_{-5}^{+6}$ & $0.44_{-7}^{+6}$ & $0.43_{-5}^{+6}$ \\
$\left\langle F_{\text {med }}-F_{\text {min }}\right\rangle$ & $0.12_{-12}^{+12}$ & $0.11_{-8}^{+7}$ & $0.15_{-7}^{+7}$ & $0.14_{-4}^{+4}$ & $0.15_{-3}^{+3}$ \\
\hline \multicolumn{2}{|l|l|l}{} & Antiperiodic boundary conditions & \\
\hline$\left\langle N_{\text {sol }} / 2\right\rangle$ & 2.7 & 7 & 20 & 93 & 717 \\
$68 \%$ CI & $2-4$ & $4-10$ & $12_{-29}$ & $42_{-165}$ & $284_{-1493}^{+3}$ \\
$\left\langle F_{\text {min }}\right\rangle$ & $0.43_{-11}^{+10}$ & $0.43_{-8}^{+7}$ & $0.41_{-7}^{+6}$ & $0.40_{-4}^{+5}$ & $0.42_{-4}^{+6}$ \\
$\left\langle F_{\text {med }}-F_{\text {min }}\right\rangle$ & $0.17_{-11}^{+21}$ & $0.15_{-9}^{+9}$ & $0.14_{-4}^{+5}$ & $0.14_{-3}^{+4}$ & $0.13_{-3}^{+2}$ \\
\hline
\end{tabular}

Table 4: Number of random orbits $N_{\text {orb }}$ and initial guesses $N_{\text {guess }}$ used for the different lattice sizes using the naive functional, together with results for periodic and antiperiodic boundary conditions. $N_{\text {sol }}$ denotes the number of minima found and $68 \%$ CI is the $68 \%$ confidence interval in the number of minima. $F_{\min }$ is the absolute minimum of the gauge fixing functional, and its median value at the local minima is $F_{\text {med }}$. The errors in the last digit(s) denote the $68 \%$ confidence interval. 